\title{
COMPUTATIONAL FLUID DYNAMICS CHALLENGES FOR HYBRID AIR VEHICLE APPLICATIONS
}

\author{
M. Carrión ${ }^{1}$, M. Biava ${ }^{1}$, R. Steijl ${ }^{1}$, G. N. Barakos ${ }^{1}$, \\ and D. Stewart ${ }^{2}$
}

${ }^{1}$ Computational Fluid Dynamics Laboratory, School of Engineering University of Glasgow James Watt South Building, Glasgow G12 8QQ, U.K. ${ }^{2}$ Hybrid Air Vehicles Ltd. 1 Hangar, Cardington Airfield, Shortstown, Bedford MK42 0TG, U.K.

\begin{abstract}
This paper begins by comparing turbulence models for the prediction of hybrid air vehicle (HAV) flows. A $6: 1$ prolate spheroid is employed for validation of the computational fluid dynamics (CFD) method. An analysis of turbulent quantities is presented and the Shear Stress Transport (SST) $k-\omega$ model is compared against a $k-\omega$ Explicit Algebraic Stress model (EASM) within the unsteady Reynolds-Averaged Navier-Stokes (RANS) framework. Further comparisons involve Scale Adaptative Simulation models and a local transition transport model. The results show that the flow around the vehicle at low pitch angles is sensitive to transition effects. At high pitch angles, the vortices generated on the suction side provide substantial lift augmentation and are better resolved by EASMs. The validated CFD method is employed for the flow around a shape similar to the Airlander aircraft of Hybrid Air Vehicles Ltd. The sensitivity of the transition location to the Reynolds number is demonstrated and the role of each vehicle's component is analyzed. It was found that the fins contributed the most to increase the lift and drag.
\end{abstract}

\section{INTRODUCTION}

Predicting the vortical flows present around lighter than air (LTA) and HAVs [1] is challenging for CFD. In the literature, prolate spheroids have been commonly used to approximate LTAs $[2,3]$, since conventional airships have ellipsoidal axisymmetric shapes [4]. The present work suggests that this is true; there are, however, substantial differences. When spheroids and airships are pitched at

(C) The authors, published by EDP Sciences. This is an Open Access article distributed under the terms of the Creative Commons Attribution License 4.0 (http://creativecommons.org/licenses/by/4.0/). 


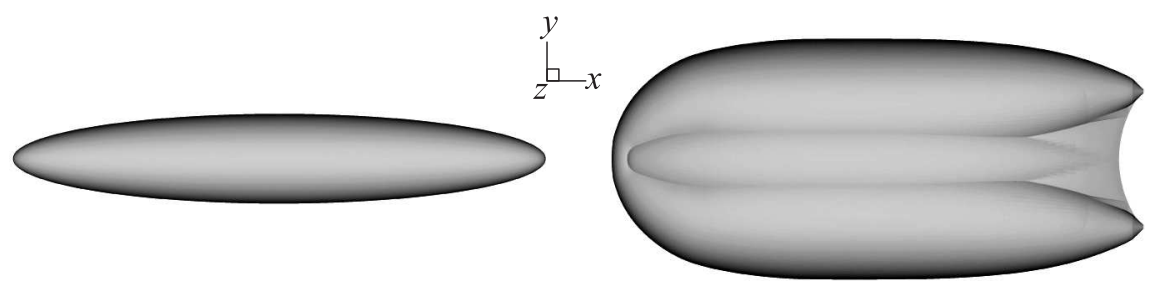

(a)

(b)

Figure 1 Comparison between a spheroid and an HAV: (a) $6: 1$ prolate spheroid; and $(b)$ hull of a representative HAV

high angles, vortices are shed due to their shape curvature and pressure gradients. In the case of spheroids, these vortices are very close to each other leading to vortical interactions further downstream. Hybrid air vehicles, on the other hand, tend to be much wider than spheroids and, especially in modern HAV designs, the hull is composed by more than one lobes to maximize the production of lift as shown in Fig. 1. This makes the distance between vortices much longer than for prolate spheroids and leads to a much weaker or even nonexistent interaction between them. In addition, airships have fins and/or strakes that fix the point of vortex formation and enhance their strength.

Computational fluid dynamics is typically based on one- or two-equation turbulence models and a Boussinesq's approximation [5]. These models assume an alignment between the Newtonian and Reynolds stress tensors. For many aerospace applications, this assumption is valid. However, for more complex problems, such as spheroids or airships, where the flows are highly threedimensional (3D) and have strong secondary flows, this assumption is very restrictive. Boussinesq models also tend to over-produce eddy viscosity near vortex cores, unless some clipping is introduced in their production terms. For these cases, more advanced turbulent models are required. One option is the use of Reynolds-stress Models (RSMs), where a transport equation is solved for each component of the Reynolds stress tensor [5]. The implementation of such models is complex and their use is costly for routine applications. Few works in the literature are found where RSMs are employed for spheroids. Alpman and Long [6] used the PUMA solver for the analysis of the flow around a $6: 1$ spheroid and a sphere. The results showed good agreement with experiments with some discrepancies in the skin friction predictions. Although the computations were costly in terms of CPU resources, the analysis of the normalized turbulent stresses in the sphere demonstrated its highly anisotropic nature and, therefore, justified the use of RSM. More recently, Yossef et al. [7] studied the spheroid at $20^{\circ}$ of pitch and $4.2 \cdot 10^{6}$ Reynolds. Boundary layer velocity components and Reynolds stresses obtained with RSM showed good agreement with the experiments [8]. 
Explicit algebraic RSMs (EARSMs) [9-11] are the nonlinear models that lay in between Boussinesq models and RSM. Explicit algebraic RSMs are based on two-equation turbulence models, but replace Boussinesq's relationship between the Reynolds stress tensor and the main strain rate with a higher-order expansion. The isotropy restriction of the Boussinesq models is, therefore, lifted. In the literature, Grudestam et al. [10] employed an EARSM for the analysis of rotating two-dimensional (2D) shear and channel flows and 3D pipe flows. Boundary layers under adverse pressure gradient, wakes, and mixing layers were studied by Hellsten [11].

There are experimental data available in the open literature for $6: 1$ prolate spheroids $[8,12,13]$ and for $3: 1$ spheroids [14]. Scott $[15,16]$ studied the $6: 1$ spheroid [12] at $20^{\circ}$ of pitch using the OVERFLOW solver. The first study [15] showed the importance of grid refinement in capturing the vortical structures. The $k-\omega$ SST model showed better pressure agreement with experiments than the $k-\omega$ and Spalart-Allmaras (SA) models. However, the skin friction was underpredicted by all the models. Detached Eddy Simulation (DES) was employed in a later study [16]; however, no improvements in the pressure and skin friction predictions were reported. This was attributed to the lack of separation of the main vortices at $20^{\circ}$ of pitch, with the DES model working as Unsteady RANS (URANS) only.

Similar DES results were obtained by Xiao et al. [17]. Although primary and secondary separations were in agreement with the experiments [12], there was no improvement in the skin friction predictions. Similar studies were performed by other authors [18-20].

Sorensen [21] also employed the $6: 1$ spheroid [12] for the validation of the $\gamma-\operatorname{Re}_{\theta}$ transition model, for Reynolds between $3.2 \cdot 10^{6}$ and $9.6 \cdot 10^{6}$, and at $0^{\circ}$ and $30^{\circ}$ of pitch. Fully turbulent and transitional cases showed good agreement with experiments for the pressure. At zero pitch, the transition model agreed better with the experimental skin friction than the fully turbulent cases, but the $\gamma-\operatorname{Re}_{\theta}$ model was not able to predict the correct location of transition at $30^{\circ}$. This was attributed to the lack of cross-flow transition prediction capability of the employed model.

Vizhinho et al. [22] developed a transition model based on SA and pretransitional quantities (V-SA). The experiments were compared against the baseline $\mathrm{SA}, \gamma-\mathrm{Re}_{\theta}$ model, and the developed V-SA transition model. The latter predicted the closest transition onset to the experiments. However, the predicted transition length was shorter than in the experiments and was attributed to an excess of turbulent kinetic energy diffusion inside the boundary layer.

The explicit algebraic model developed by Rumsay and Gatski [23] was employed by Morrison et al. [24] for the analysis of a $6: 1$ spheroid at $30^{\circ}$ of pitch and at Reynolds of $43 \cdot 10^{6}$. Better agreement in the pressure predictions was obtained with the EASM than with the $k-\omega$ model [25]. However, lack of grid convergence was reported and skin friction predictions were not investigated. 
This paper explores different turbulence models aiming to find the best framework to model airship-like flows. A study of the aerodynamics for a variant of a HAV is then provided.

\section{NUMERICAL METHOD}

\subsection{HMB2 Flow Solver}

The Helicopter Multi-Block (HMB2) CFD solver [26] is used for the present work and has so far been validated for a number of applications, including helicopters, wind turbines, turboprop, and UCAV (unmanned combat aerial vehicle) aircraft. HMB2 solves the Navier-Stokes equations in integral form using the Arbitrary Lagrangian Eulerian formulation for time-dependent domains with moving boundaries:

$$
\frac{d}{d t} \int_{V(t)} \overrightarrow{\mathbf{w}} d V+\int_{\partial V(t)}\left(\vec{F}_{i}(\overrightarrow{\mathbf{w}})-\vec{F}_{v}(\overrightarrow{\mathbf{w}})\right) \vec{n} d S=\vec{S}
$$

where $V(t)$ is the time-dependent control volume; $\partial V(t)$ is its boundary; $\overrightarrow{\mathbf{w}}$ is the vector of conserved variables $[\rho, \rho u, \rho v, \rho w, \rho E]^{\mathrm{T}}$; and $\vec{F}_{i}$ and $\vec{F}_{v}$ are the inviscid and viscous fluxes, including the effects of the mesh movement. The NavierStokes equations are discretised using a cell-centered finite-volume approach, leading to the following equation:

$$
\frac{\partial}{\partial t}\left(\mathbf{w}_{i, j, k} V_{i, j, k}\right)=-\mathbf{R}_{i, j, k}\left(\mathbf{w}_{i, j, k}\right)
$$

where $i, j$, and $k$ are the cell indices; $\mathbf{w}$ are the variables; $\mathbf{R}$ are the residuals; and $V$ is the volume. The upwind scheme [27] is used for the discretization of the convective terms and MUSCL (monotone upstream scheme for conservation laws) [28] variable extrapolation is used to provide formally 3rd-order accuracy. To account for low-speed flows, the low-Mach Roe scheme of Rieper [29] is employed [30]. The linearized system is solved using the generalised conjugate gradient with a block incomplete lower-upper preconditioner [31].

\subsection{Turbulence Modeling}

Fully turbulent flows are usually assumed in aerospace applications; however, some problems involve both laminar and turbulent flows that need to be correctly captured. For this type of problems where the effect of transition is important, transition models such as $\gamma-\operatorname{Re}_{\theta_{t}}$ should be employed. On the other hand, 
EASMs (EARSMs) provide good prediction of the vortex cores. In addition, unsteady problems with large separation can be predicted with Scale-Adaptive Simulation (SAS). The HMB2 solver has a library of turbulence closures which includes several one- and two-equation turbulence models and versions of the $k-\omega$ model, including the SAS model [32], the $k-\omega$ EASM (EARSM) [11], and the $\gamma-\operatorname{Re}_{\theta_{t}}$ transition model [33].

\subsubsection{Explicit algebraic stress $k-\omega$ model}

The following are the equations for the $k-\omega$ baseline (BSL) model of Menter [34]. In the EARSM model from Hellsten [11], the production term $(P)$ and the eddy viscosity $\left(\mu_{t}\right)$ have additional nonlinear contributions:

$$
\begin{aligned}
& \frac{\partial(\rho k)}{\partial t}+\frac{\partial\left(\rho u_{j} k\right)}{\partial x_{j}}=P-\beta^{*} \rho \omega k+\frac{\partial}{\partial x_{j}}\left(\left(\mu+\sigma_{k} \mu_{t}\right) \frac{\partial k}{\partial x_{j}}\right) ; \\
& \frac{\partial(\rho \omega)}{\partial t}+\frac{\partial\left(\rho u_{j} \omega\right)}{\partial x_{j}}=\frac{\gamma \omega}{k} P-\beta \rho \omega^{2}+\frac{\partial}{\partial x_{j}}\left(\left(\mu+\sigma_{\omega} \mu_{t}\right) \frac{\partial \omega}{\partial x_{j}}\right) \\
& +\sigma_{d} \frac{\rho}{\omega} \max \left(\frac{\partial k}{\partial x_{k}} \frac{\partial \omega}{\partial x_{k}}, 0\right) .
\end{aligned}
$$

The terms affected in the production $(P)$ are the viscous stresses $\left(\tau_{i j}\right)$, as presented in

$$
P=\tau_{i j} \frac{\partial u_{i}}{\partial x_{j}}
$$

Here,

$$
\tau_{i j}=2 \mu_{t}\left(\frac{1}{2}\left(\frac{\partial u_{i}}{\partial x_{j}}+\frac{\partial u_{j}}{\partial x_{i}}\right)-\frac{1}{3} \frac{\partial u_{k}}{\partial x_{k}} \delta_{i j}\right)-\frac{2}{3} \rho k \delta_{i j}-a_{i j}^{(\mathrm{ex})} \rho k
$$

where

$$
\mu_{t}=\frac{C_{\mu}}{\beta^{*}} \frac{\rho k}{\omega}
$$

One contribution comes from the addition of the anisotropic terms $\left(a_{i j}^{(\mathrm{ex})}\right)$ whose expression is presented in

$$
\begin{aligned}
& a_{i j}^{(\mathrm{ex})}=\beta_{3}(\left.\Omega_{i k}^{*} \Omega_{k j}^{*}-\frac{1}{3} I I_{\Omega} \delta_{i j}\right)+ \\
&+\beta_{4}\left(S_{i k} \Omega_{k l}^{*} \Omega_{l j}^{*}+\Omega_{i k}^{*} \Omega_{k l}^{*} S_{l j}-I I_{\Omega} S_{i j}-\frac{2}{3} I V \delta_{i j}\right) \\
&+\beta_{9}\left(\Omega_{i k}^{*} S_{k l} \Omega_{l m}^{*} \Omega_{m j}^{*}-\Omega_{i k}^{*} \Omega_{k l}^{*} S_{l m} \Omega_{m j}^{*}\right) .
\end{aligned}
$$


The other contribution comes from the scaling of the eddy viscosity $\left(\mu_{t}\right)$ by a nonlinear coefficient $C_{\mu}$, given in

$$
C_{\mu}=-\frac{1}{2}\left(\beta_{1}+I I_{\Omega} \beta_{6}\right) .
$$

In this model, $\beta^{*}=0.09$, like in the BSL model [34]. The closure coefficients $\gamma, \beta, \sigma_{k}, \sigma_{\omega}$, and $\sigma_{d}$ are obtained using the following equation with the blending function $f_{b}$ :

$$
C=f_{b} C_{1}+\left(1-f_{b}\right) C_{2}, \quad f_{b}=\tanh \left(1.5 \Gamma^{4}\right) .
$$

Here,

$$
\Gamma=\min \left[\max \left(\Gamma_{1}, \Gamma_{2}\right), \Gamma_{3}\right]
$$

where

$$
\begin{gathered}
\Gamma_{1}=\frac{\sqrt{k}}{\beta^{*} \omega d} ; \quad \Gamma_{2}=\frac{500 \nu}{\omega d^{2}} ; \\
\Gamma_{3}=\frac{20 k}{\max \left(\left(d^{2} / \omega\right)\left(\left(\partial k / \partial x_{k}\right)\left(\partial \omega / \partial x_{k}\right)\right), 200 k_{\infty}\right)} .
\end{gathered}
$$

Note that the terms $\Gamma_{1}$ and $\Gamma_{2}$ are exactly the same as the ones in the BSL model. The coefficients for the blending function are detailed in [11].

\subsubsection{Scale-adaptive simulation}

Scale-adaptive simulation [32] is a modification of an SST-RANS model [34] based on the use of the second mechanical scale (in the form of second derivatives of velocity) in the source terms of the underlying turbulence model. An additional term $Q_{\mathrm{SAS}}$ is then added in the transport equation for the specific dissipation rate $\omega$,

$$
Q_{\mathrm{SAS}}=\max \left[\rho \zeta_{2} \kappa S^{2}\left(\frac{l}{l_{v K}}\right)-C \frac{2 \rho k}{\sigma_{\Phi}} \max \left(\frac{|\nabla \omega|^{2}}{\omega^{2}}, \frac{|\nabla k|^{2}}{k^{2}}\right), 0\right]
$$

where $l$ is the length scale; $l_{v K}$ is the von Karman length scale in classic boundary layer definition; and the constants $\zeta_{2}=3.51, \sigma_{\Phi}=2 / 3$, and $C=2$.

Scale-adaptive simulation is a hybrid RANS / Large-Eddy Simulation (LES) model which can produce spectral content for unstable flows, adjusting the turbulence length scale to the local flow inhomogeneities and balancing the contributions of modeled and resolved parts of the turbulent stresses. For steady flows, it acts as a RANS model and for flows with transient instabilities like those with massive separation, the model reduces its eddy viscosity according to the locally resolved vortex size represented by the von Karman length scale. The SAS model can resolve the turbulent spectrum down to the grid limit and avoids RANS-typical single-mode vortex structure. 


\subsubsection{Local correlation-based transition model}

The $\gamma-\operatorname{Re}_{\theta}$, or Local Correlation-based Transition Model (LCTM) [35], uses two additional transport equations: one for intermittency $(\gamma)$ and one for the transition momentum thickness Reynolds number $\left(\operatorname{Re}_{\theta t}\right)$, which is formulated in terms of the scalar quantity, $\widetilde{\operatorname{Re}_{\theta t}}$. The $\gamma$-equation is used to trigger the transition process and the $\widetilde{R e}_{\theta t}$-equation is employed to avoid nonlocal variables and pass information from the freestream to the boundary layer. The transport equations are:

$$
\begin{aligned}
\frac{\partial(\rho \gamma)}{\partial t}+\frac{\partial \rho u_{j} \gamma}{\partial x_{j}} & =P_{\gamma 1}+P_{\gamma 2}+\frac{\partial}{\partial x_{j}}\left[\left(\mu+\frac{\mu_{t}}{\sigma_{f}}\right) \frac{\partial \gamma}{\partial x_{j}}\right] ; \\
\frac{\partial\left(\rho \widetilde{\operatorname{Re}}_{\theta t}\right)}{\partial t}+\frac{\partial\left(\rho u_{j} \widetilde{\operatorname{Re}}_{\theta t}\right)}{\partial x_{j}} & =P_{\theta t}+\frac{\partial}{\partial x_{j}}\left[\sigma_{\theta t}\left(\mu+\mu_{t}\right) \frac{\partial \widetilde{\operatorname{Re}}_{\theta t}}{\partial x_{j}}\right]
\end{aligned}
$$

where $\rho$ is the density; $u_{j}$ is the velocity vector; $\mu$ is the molecular viscosity; and $\mu_{t}$ is the eddy viscosity. The production terms, $P_{\gamma 1}, P_{\gamma 2}$, and $P_{\theta t}$ are defined as follows:

$$
\begin{aligned}
P_{\gamma 1} & =\left(1-c_{\epsilon 1} \gamma\right) c_{\alpha 1} F_{\text {length }} \rho S\left(\gamma F_{\text {onset }}\right)^{c_{\alpha}} ; \\
P_{\gamma 2} & =\left(1-c_{\epsilon 2} \gamma\right) c_{\alpha 2} \rho \Omega \gamma F_{\text {turb }} ; \\
P_{\theta t} & =c_{\theta t} \frac{(\rho U)^{2}}{500 \mu}\left(\operatorname{Re}_{\theta t}-\widetilde{\operatorname{Re}}_{\theta t}\right)\left(1.0-F_{\theta t}\right)
\end{aligned}
$$

where $\Omega$ is the vorticity magnitude; $S$ is the strain rate magnitude; $U$ is the local velocity magnitude; the parameters $F_{\text {length }}$ and $F_{\text {onset }}$ are used to control the length and onset location of transition, respectively; and $F_{\text {turb }}$ and $F_{\theta t}$ are the parameters for controlling the destruction/relaminarization of the boundary layer and the boundary layer detector, respectively. Term $P_{\gamma 2}$ acts as a sink and ensures that the intermittency remains close to zero in the laminar boundary layer. The production term $P_{\theta t}$ in Eq. (3) is designed to force the transported scalar $\widetilde{\operatorname{Re}}_{\theta t}$ in the freestream to match the local value of $\operatorname{Re}_{\theta t}$ into the boundary layer. Parameter $\operatorname{Re}_{\theta t}$ is calculated locally from the empirical correlation proposed by Menter et al. [35]. This parameter is the critical Reynolds number where the intermittency first starts to increase in the boundary layer. This occurs upstream of the transition Reynolds number. Likewise, $\widetilde{\operatorname{Re}_{\theta t}}$ is the location where the velocity profile begins to deviate from the purely laminar profile.

The boundary condition for the intermittency factor $(\gamma)$ at the freestream and wall boundaries is zero flux. For $\tilde{R e}_{\theta t}$, the boundary condition at the freestream and wall boundaries is also zero flux. Finally, the model constants are $c_{a 1}=2$; $c_{e 1}=1 ; c_{a 2}=0.06 ; c_{e 2}=50 ; c_{a}=0.5 ; \sigma_{\gamma}=1 ; \sigma_{\theta t}=2$; and $c_{\theta t}=0.03$. 


\section{MESH GENERATION}

Multiblock structured topologies were employed to allow for good representation of the spheroid and airship surfaces. The blocks are also used for easy sharing of the computational load between parallel processors.

As shown in Fig. 2, an O-topology was employed for the $6: 1$ prolate spheroid grid, with 576 blocks for good load balancing between processors. The inflow, outflow, and far-field boundaries extended $10 L$ from the spheroid surface where $L$ is the length of the spheroid. Since the spheroid is symmetric with respect to its long axis, only half of it was meshed. A fine grid was employed for good boundary layer resolution and for capturing the vortices found above the spheroid. Hence, the off-body grid was kept uniform in the region where vortices were expected, with maximum cell sizes of $0.03 \% L$ (Fig. $2 b$ ). Good grid resolution close to the wall is also a requirement for the $\gamma-\operatorname{Re}_{\theta}$ turbulence model. In the first block normal to the surface, 74 cells were used, with a first cell spacing of $10^{-5} \mathrm{~L}$ and an expansion ratio of 1.05 , to ensure $y^{+}<1$. Around the spheroid azimuth, 480 cells were employed and 592 cells were used along its long axis, with a maximum cell

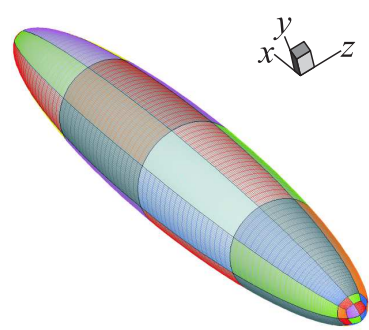

(a)

(c)

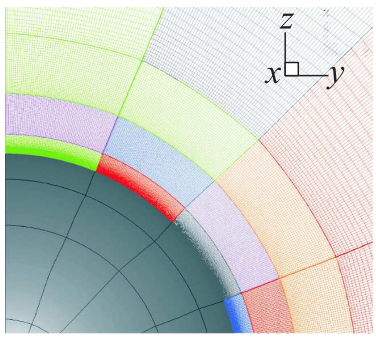

(b)

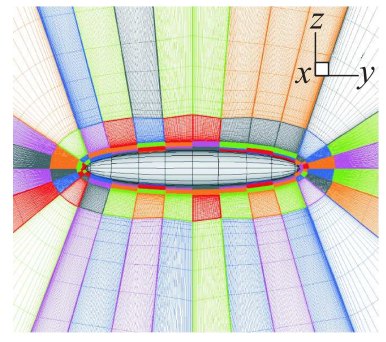

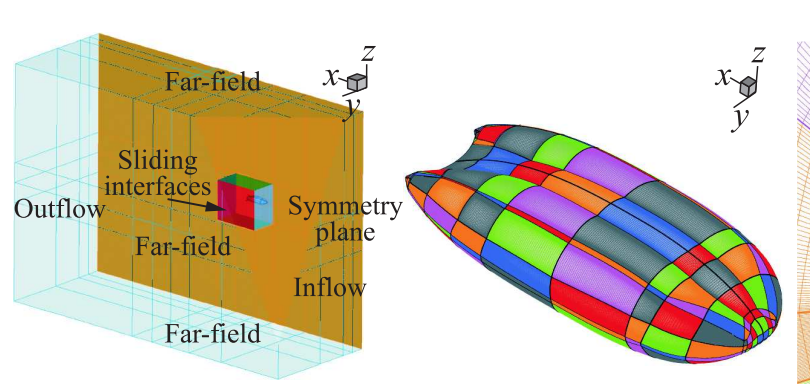

(d)

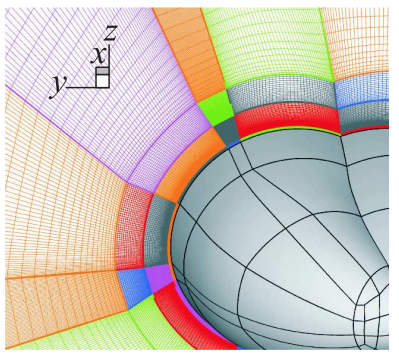

(e)

Figure 2 Multiblock mesh for $6: 1$ prolate spheroid and HAV's bare hull: $(a)$ spheroid grid surface; (b) O-topology around the spheroid; (c) HAV boundaries; (d) HAV's grid surface (AL 1); and (e) slice through the HAV's grid 


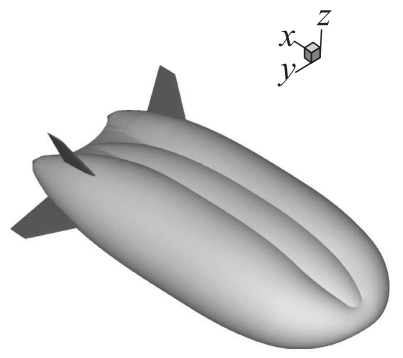

(a)

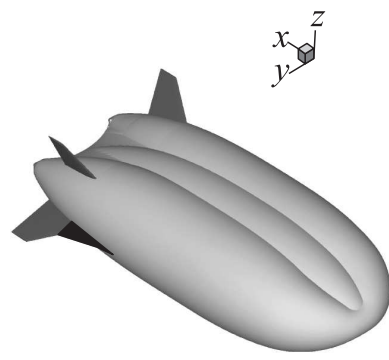

(b)

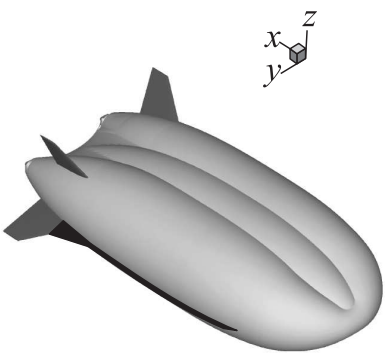

(c)

Figure 3 Vehicle configurations: (a) AL 2: hull \& fins; (b) AL 3: hull, fins, and LERX; and (c) AL 4: hull, fins, LERX, and strakes

size in that direction of $0.003 \mathrm{~L}$. This led to a 40 -million cells grid. Assuming symmetry, only half of the HAV's hull was meshed. At the inflow, outflow, and far-field boundaries, freestream conditions were imposed. Five sliding planes [36] were employed to allow for localized grid refinement near the body and to have a cartesian grid in the rest of the domain. These planes are the ones close to the body shown in Fig. 2c. An O-topology was employed around the HAV for optimal orthogonality to the surface (Fig. 2e). The first cell size of $3 \mu \mathrm{m}$ was employed to ensure $y^{+}<1$ and 50 cells were used to capture the boundary layer. Around the hull, 516 cells were employed and 362 cells along its length. The grid had a total of $31 \cdot 10^{6}$ cells.

Four configurations were considered to study the role of the components of an approximated hybrid air vehicle (Fig. 3). Configuration AL $\mathbf{1}$ consists of the bare hull; the fins are then added (AL 2) and also the leading edge extension (LERX) in configuration AL 3. Finally, strakes are considered in configuration AL 4. To assess grid convergence, two grid levels were employed for the baseline case (grids AL 4 and AL 5).

\section{RESULTS AND DISCUSSION}

In this section, the computational results are presented. For the 6:1 spheroid computations, a Reynolds number of $4.2 \cdot 10^{6}$ (based on the spheroid's length $L$ ) and Mach number of 0.15 were employed. In addition, the spheroid was pitched at $20^{\circ}$. For the analysis of the HAV hull, a Reynolds number of $3 \cdot 10^{6}$ (based on the vehicle's length) and a Mach of 0.12 were selected. The HAV was pitched at $20^{\circ}$ and $30^{\circ}$. Further computations are then presented for the approximate HAV (AL), including the role of the strakes in the aerodynamic coefficients and effect of the flow conditions and Reynolds number on the transition onset. For 
Table 1 Summary of test cases

\begin{tabular}{ccccr}
\hline Case & Geometry & Size $($ Blocks) & Turbulence model & CPUs \\
\hline SPH.1 & $6: 1$ Spheroid & $40 \cdot 10^{6}(576)$ & URANS SST & 128 \\
SPH.2 & $6: 1$ Spheroid & $40 \cdot 10^{6}(576)$ & URANS BSL + EARSM & 128 \\
SPH.3 & $6: 1$ Spheroid & $40 \cdot 10^{6}(576)$ & SAS SST & 128 \\
SPH.4 & $6: 1$ Spheroid & $40 \cdot 10^{6}(576)$ & URANS $\gamma-\operatorname{Re}_{\theta}$ & 128 \\
HAV.1 & Hybrid air vehicle & $31 \cdot 10^{6}(1308)$ & URANS SST & 128 \\
HAV.2 & Hybrid air vehicle & $31 \cdot 10^{6}(1308)$ & URANS BSL + EARSM & 128 \\
\hline AL 1 & Bare hull & $9.2 \cdot 10^{6}(1069)$ & URANS SST & 64 \\
AL 2 & Hull and fins & $9.5 \cdot 10^{6}(1151)$ & URANS SST & 64 \\
AL 3 & Hull, fins, and LERX & $10.8(1203)$ & URANS SST & 64 \\
AL 4 Hull, fins, LERX, and strakes & $10.8(1203)$ & URANS SST & 64 \\
AL 5 & Refined AL 4 & $10.8(1203)$ & URANS SST & 64 \\
\hline
\end{tabular}

these cases, a wind speed of $40 \mathrm{~m} / \mathrm{s}$ was employed and sea level conditions were assumed. Unless otherwise specified, the Reynolds number, based on the vehicle's length $(L)$, was $3 \cdot 10^{6}$. The RANS and URANS computations were performed, with the $k-\omega$ SST turbulence model by Menter [34]. Table 1 provides a summary of the test cases, including the employed grids, turbulence model, and the number of CPUs used in the computations. The first computations were performed for the study of different turbulence modeling frameworks. The last 5 sets of computations were performed for the analysis of the components of the HAV.

The spheroid flow at high pitch is characterized by the development of vortices due to curvature effects. Figure 4 presents a schematic of the flow direction and
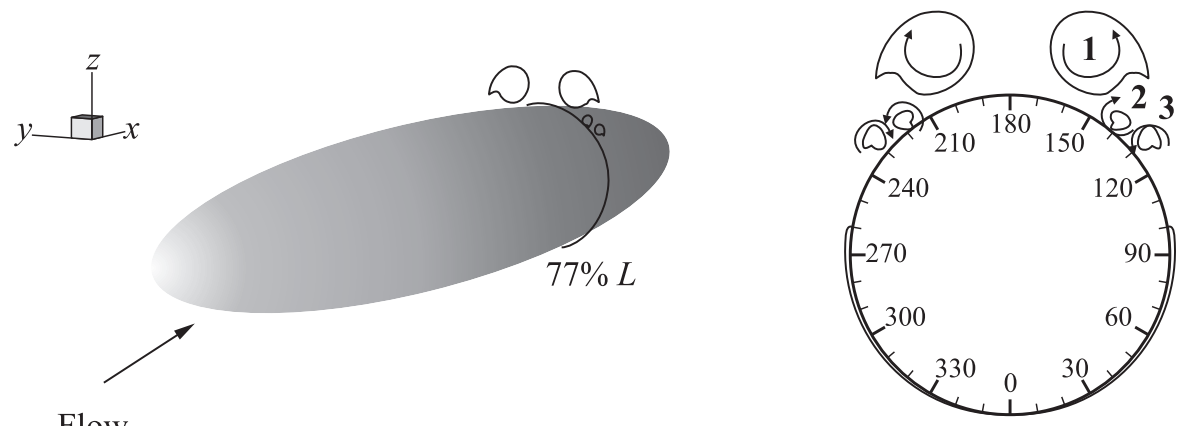

Flow

Figure 4 Schematic of vortices generated around the $6: 1$ prolate spheroid at $20^{\circ}$ of pitch 
the vortices created at $77 \% \mathrm{~L}$. A primary vortex $(\mathbf{1})$ is created at an azimuth of $\varphi=160^{\circ}$. Secondary (2) and tertiary (3) vortices also appear in this type of flow, located at $\varphi=140^{\circ}$ and $130^{\circ}$, respectively. Indication of their rotation direction (as seen from the front looking downstream) is included.

\subsection{Validation of the Method}

The HMB2 method is first validated with the pressure measurements performed by Wetzel [12] and the friction measurements by Chesnakas et al. [13]. In the experiments [12], the spheroid was tripped at $20 \% \mathrm{~L}$. Pressure taps were installed and the model was rolled to sweep the transducers from the windward to the leerward sides and to map out the pressure distribution over the model surface. Data were taken only for one half of the model, assuming symmetry of the flow. The skin friction data were not measured directly; the velocity profiles were measured and fitted to a Spalding-type wall law [13].

Figure 5 shows pressure and friction coefficients around the spheroid azimuth, through a slice at $x=77 \% L$. The CFD results $(k-\omega$ SST [34], $k-\omega$ BSL + EARSM [11], and $k-\omega-\gamma-\operatorname{Re}_{\theta}$ transition model [35]) and experiments [12, 13] are compared. The DES results presented by Constantinescu et al. [19] are also included. The SST computation was performed using 40- and 12-million cells grids. The solutions on both grids are practically identical and, therefore, grid independence was assessed in terms of pressure and friction coefficients. For the rest of the paper, however, the fine grid was employed as it enabled better resolution of the vortices off-body.

The presence of the main vortex $(\mathrm{V1})$ at $\varphi=160^{\circ}$ very close to the spheroid surface leads to a drop in pressure as can be observed in Fig. $5 a$, which is in

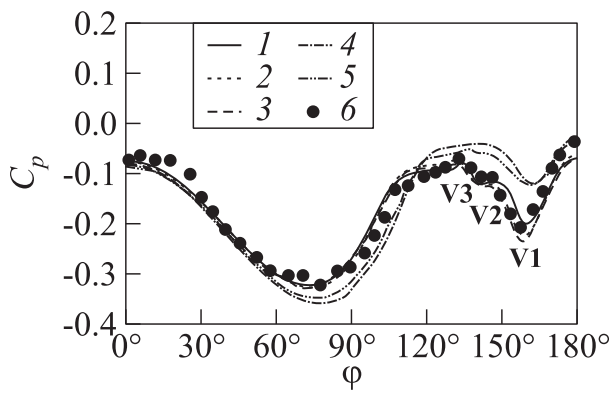

(a)

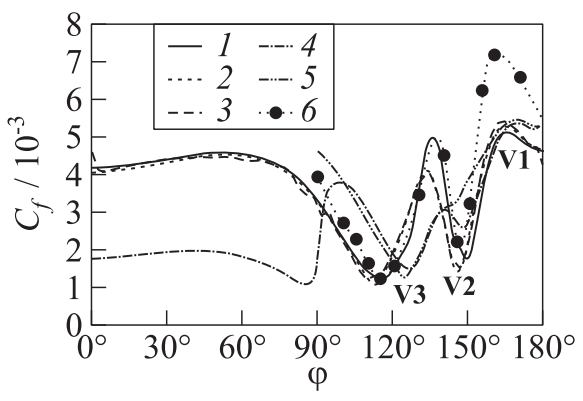

(b)

Figure 5 Spheroid's azimuthal pressure $(a)$ and friction coefficients $(b)$ at $77 \% \mathrm{~L}$ at $\operatorname{Re}=4.2 \cdot 10^{6}, \mathrm{M}=0.15$, and $\alpha=20^{\circ}: 1-k-\omega \mathrm{BSL}+\mathrm{EARSM} ; 2-k-\omega \mathrm{SST}$ $\left(40 \cdot 10^{6}\right) ; 3-k-\omega \operatorname{SST}\left(12 \cdot 10^{6}\right) ; 4-k-\omega-\gamma-\operatorname{Re}_{\theta} ; 5-[19]$; and $6-$ experiments 
very good agreement with experimental data [12]. A small drop in pressure is also observed at $\varphi=140^{\circ}$, corresponding to the secondary vortex (V2), and a small peak at $130^{\circ}$ of azimuth, where the tertiary vortex (V3) is located. The differences between the SST and the EARSM models are very small in the lower surface of the spheroid (from $0^{\circ}$ to $90^{\circ}$ ). Once the vortices are developed, the EARSM improves the agreement with experiments. The transitional model, however, presents poor agreement with experiments. This is expected, since, during the experiments, the spheroid was tripped at $20 \% L$ and at this location, the boundary layer was fully turbulent. The $k-\omega-\gamma-\operatorname{Re}_{\theta}$ model predicts free transition and at this axial location, parts of the boundary layer are laminar. The behavior of this model is further discussed in subsection 4.5.

For the friction coefficient $\left(C_{f}\right)$ presented in Fig. $5 b$, a much better agreement with the experiments [13] was obtained with the EARSM, mainly in the region where the secondary (V2) and tertiary (V3) vortices are created at $\varphi=140^{\circ}$ and $130^{\circ}$, respectively. Note that this prediction is much closer to the experiments than the one reported by Constantinescu et al. [19]. At azimuth of $160^{\circ}$, where the main vortex $(\mathbf{V 1})$ is located, all models underpredict the peak in $C_{f}$. This peak underprediction was also reported in $[15,16,19]$, even though they employed different turbulence models (RANS and DES with the SA model). These discrepancies in friction could be due to difficulties of the turbulence models to correctly predict this vortical structure, which seem to be weaker in the CFD, but also difficulties in the experiment to measure these quantities in the wind tunnel with high level of accuracy. Computations on a full spheroid without symmetry condition did not improve this aspect of the comparison. Much the same way, different laws for evaluating the $C_{f}$ including fits to velocity with polynomials showed little sensitivity due to the employed fine mesh.

\subsection{Analysis of the Stress Tensor}

To better understand the mechanism of the EARSM, this subsection provides an overview of the components of the turbulent stress tensor and a comparison with the $k-\omega$ SST model. As presented in subsection 4.1, the EARSM improved the agreement with the experiments with respect to $k-\omega \mathrm{SST}$, due to a reduction of the eddy viscosity in the vortex core. This can be seen in Fig. 6 that compares the turbulent eddy viscosity $\left(\operatorname{Re}_{T}=\mu_{T} / \mu\right)$ through a slice at $x=0.77 \% \mathrm{~L}$. As can be seen, at the edge of the primary vortex, the eddy viscosity levels are approximately the same for both turbulence models. On the other hand, a strong reduction in the eddy viscosity at the core is observed in the EARSM (Fig. 6c). This reduction of the turbulence levels is due to the nonlinear factor $\left(C_{\mu} / \beta^{*}\right)$ that scales the turbulent eddy viscosity, depending on the local shear and vorticity, as was shown in Eq. (2). For the same axial slice through the main vortex, Fig. $6 d$ shows the distribution 


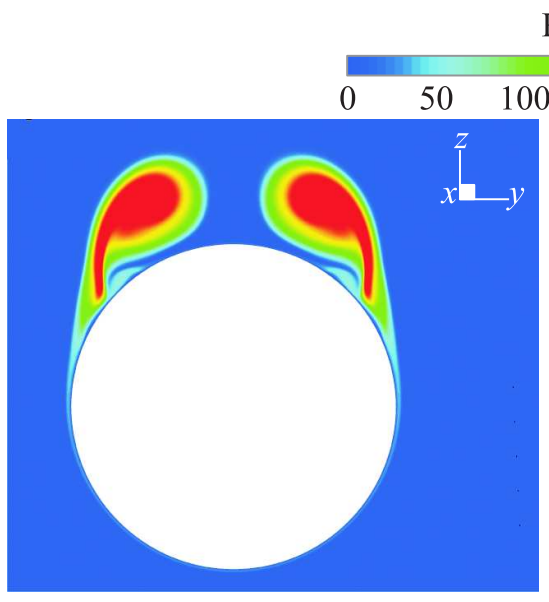

(a)

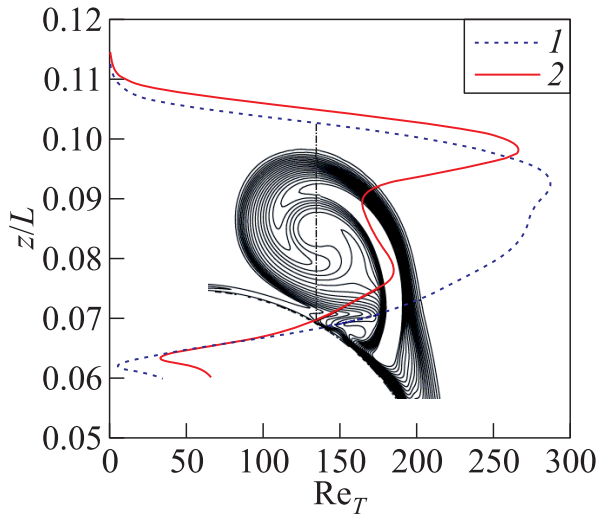

(c)

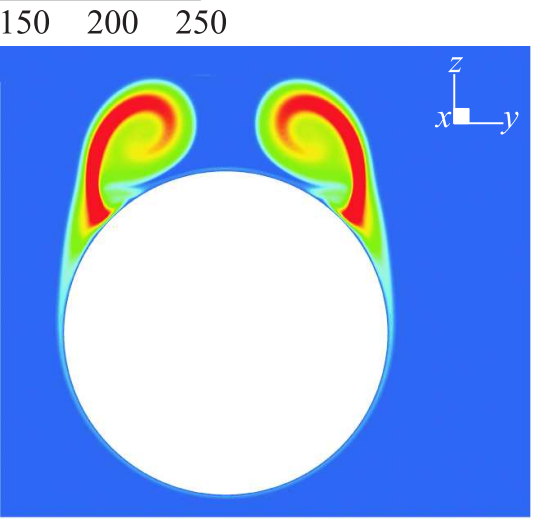

(b)
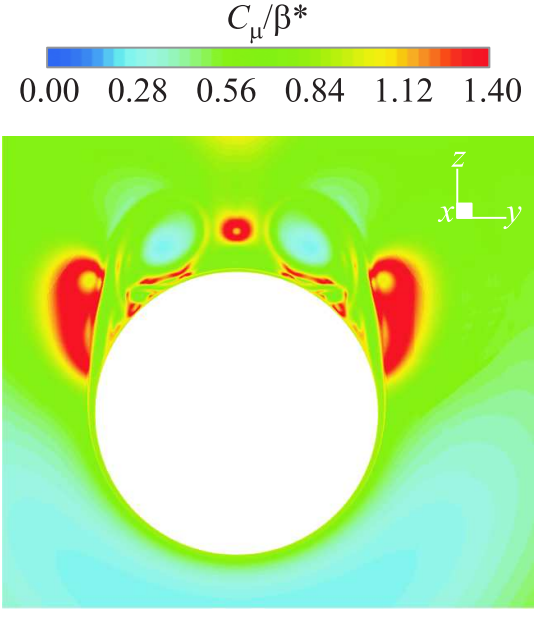

(d)

Figure 6 Turbulent eddy viscosity $\left(\operatorname{Re}_{T}\right)$ and $C_{\mu} / \beta^{*}$ at $x=0.77 \% L, \operatorname{Re}=4.2 \cdot 10^{6}$, $\mathrm{M}=0.15$, and $\alpha=20^{\circ}:$ (a) $k-\omega \mathrm{SST} ;(b) k-\omega \mathrm{BSL}+\mathrm{EARSM} ;(c)$ line through the vortex. Isolines of $\operatorname{Re}_{T}$ are shown $(1-k-\omega \mathrm{SST}$; and $2-k-\omega \mathrm{BSL}+\mathrm{EARSM})$; and (d) $C_{\mu} / \beta^{*}$ distribution $(k-\omega \mathrm{BSL}+\mathrm{EARSM})$

of the $C_{\mu} / \beta^{*}$ scaling factor where the eddy viscosity at the core is completely eliminated.

In the EARSM, the stress tensor is defined as

$$
\tau_{i j}=\tau_{i j}^{*}-a_{i j}^{(\mathrm{ex})} \rho k
$$


where $\tau_{i j}^{*}$ is the Boussinesq stress tensor but employing the nonlinear turbulent viscosity shown in Eq. (1) and $a_{i j}^{(\mathrm{ex})}$ is the anisotropy tensor. This is a symmetric matrix, whose components are shown in Fig. 7, with contours on a slice through the main vortex (at $x=77 \% L$ ). Note that $a_{i j}^{(\mathrm{ex})}$ is scaled with $1 /\left(\beta^{*} \omega\right)$.

Figure 8 shows the first three invariants of the two contributions of the total stress tensor of Eq. (4): the stress contribution $\left(\tau_{i j}^{*}\right)$ and the anisotropy contribution $\left(a_{i j}^{(e x)} \rho k\right)$. As can be seen in Figs. $8 a$ and $8 d$, the first invariant of the anisotropy tensor $\left(I_{a}=\operatorname{tr}(a)\right)$ is practically zero and, therefore, the main contribution to the stress tensor comes from $\tau_{i j}^{*}$. The second, $I I_{a}=$ $(1 / 2)\left(\operatorname{tr}(a)^{2}-\operatorname{tr}(a \cdot a)\right)$, and the third, $I I I_{a}=\operatorname{det}(a)$, invariants show that the anisotropy is normal to the viscous stress as for the same cell, each contribution is of the opposite sign.

A comparison of the cross terms of the stress tensor between the EARSM and $k-\omega$ SST models is presented in Fig. 9. The values were extracted along two circular lines: one passing through the main vortex (see Fig. 9, left column) and another line through the core of the secondary vortex (see Fig. 9, right column). As can be observed, the peaks are located at the same azimuthal position for both models $\left(\varphi=140^{\circ}\right.$ for the secondary vortex and $\varphi=160^{\circ}$ for the main vortex). In general, larger amplitudes are observed in the EARSM that seems to capture more spatial variations than the $k-\omega$ SST. This can be seen in Fig. 9, right column.

\subsection{Comparison between URANS-EARSM and SAS-SST models}

The URANS results employing the SST model [34] and EARSM [11] showed that the later was able to capture better the vortices generated around spheroids, leading to a better agreement with the experimental data. This subsection aims to make a comparison between the URANS-EARSM and SAS-SST [32] computations.

Figure 10 shows the slices of pressure and friction coefficients at $x=0.77 \% \mathrm{~L}$. For the SAS-SST model, two time-step sizes were employed $\left(\Delta t^{*}=0.02\right.$ and 0.005 ) and identical solutions were obtained, showing independence on the size of the time step. The SAS-SST model did not improve the agreement with the experiments unlike the EARSM model. This is due to the fact that the SAS model reduced the overall eddy viscosity on the vortex but not locally at the core. This can be observed if the turbulence levels of the two models are compared (see Figs. $6 b$ and 11a). A line crossing the main vortex (Fig. 11b) shows that the eddy viscosity at the edge of the vortex is higher for the EARSM model which leads to stronger vortices. These results show that to predict this flow, accounting for anisotropy is essential. 


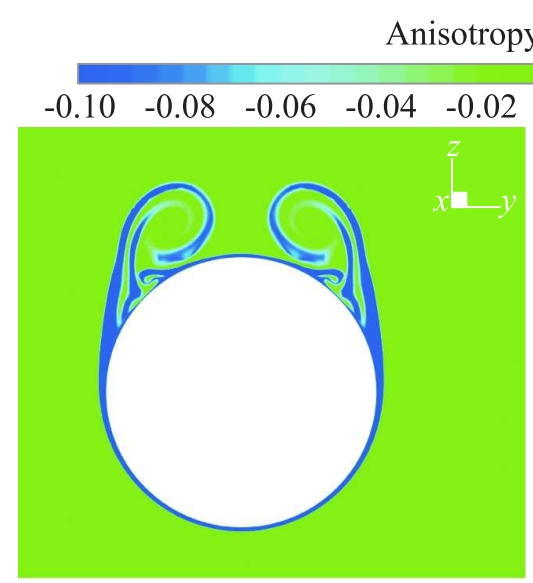

(a)

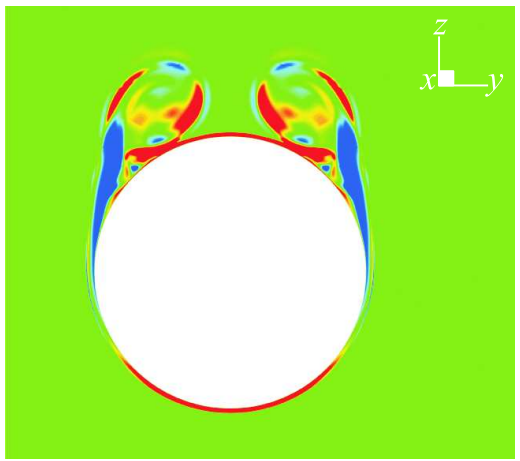

(c)

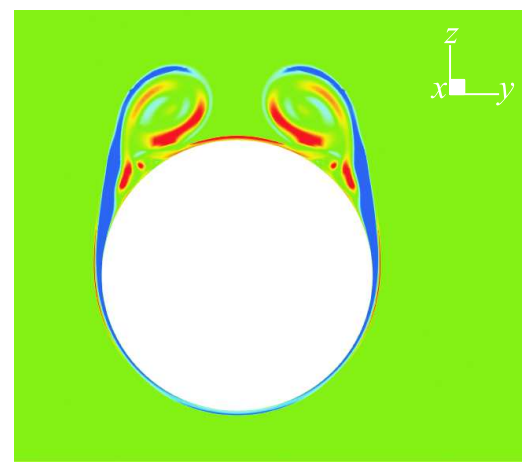

(e)

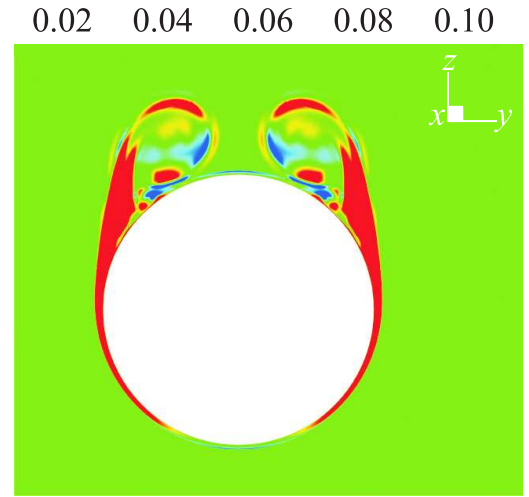

(b)

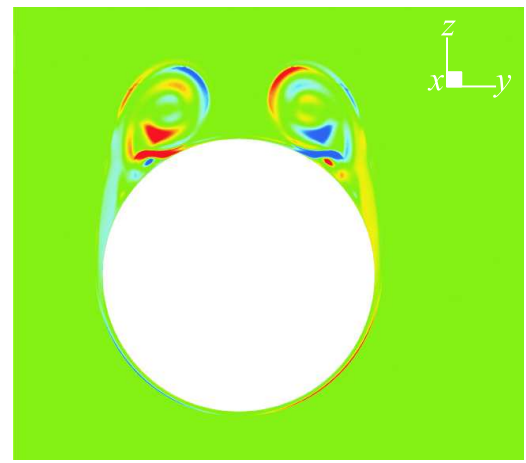

(d)

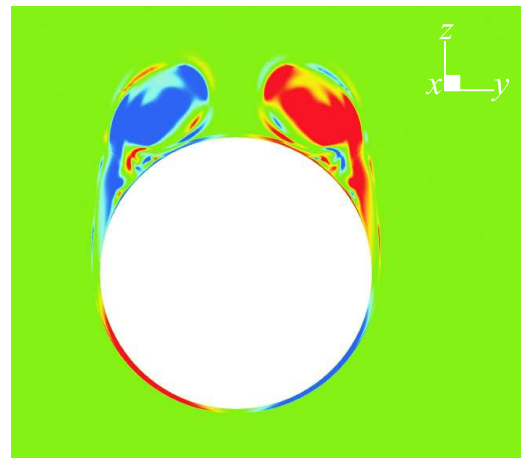

$(f)$

Figure 7 Anisotropy coefficients of the EARSM around the main vortex $(x$ $=0.77 \% L)$ at $\operatorname{Re}=4.2 \cdot 10^{6}, \mathrm{M}=0.15$, and $\alpha=20^{\circ}:(a) a_{x x}^{(\mathrm{ex})} ;(b) a_{y y}^{(\mathrm{ex})} ;(c) a_{z z}^{(\mathrm{ex})}$; (d) $a_{x y}^{(\mathrm{ex})}=a_{y x}^{(\mathrm{ex})} ;(e) a_{x z}^{(\mathrm{ex})}=a_{z x}^{(\mathrm{ex})} ;$ and $(f) a_{y z}^{(\mathrm{ex})}=a_{z y}^{(\mathrm{ex})}$ 


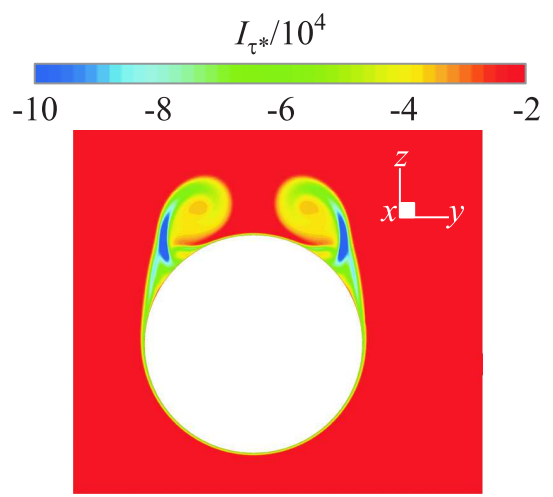

(a)

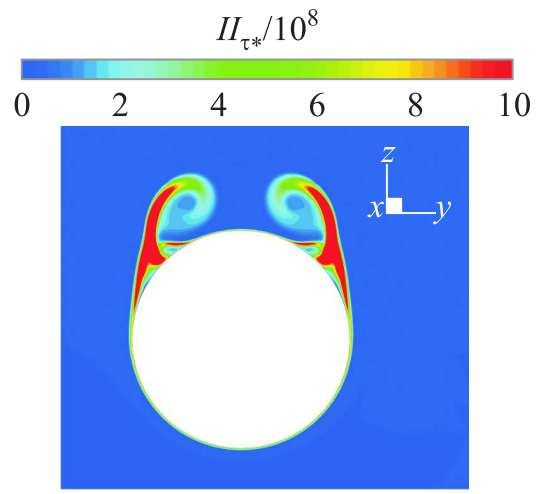

(b)

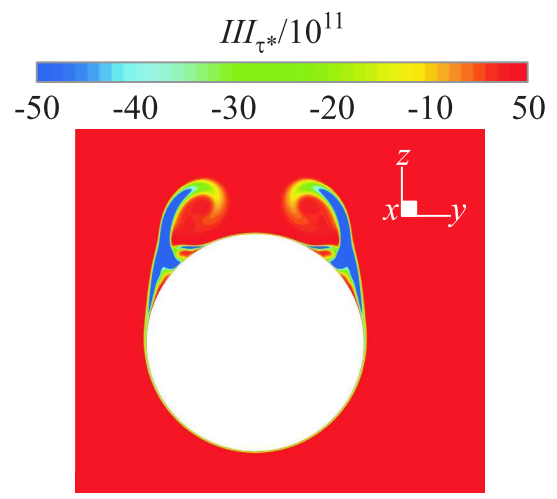

(c)

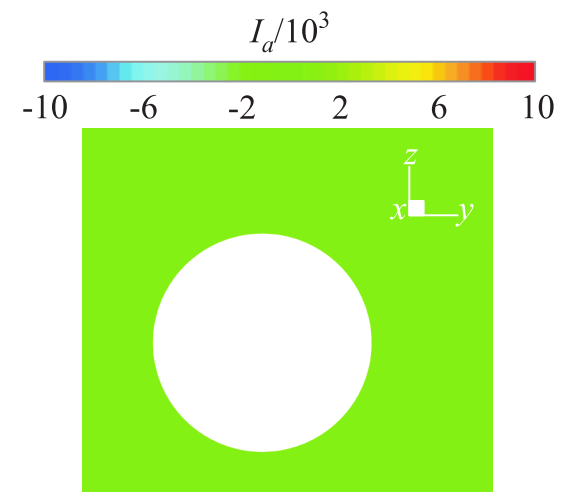

(d)

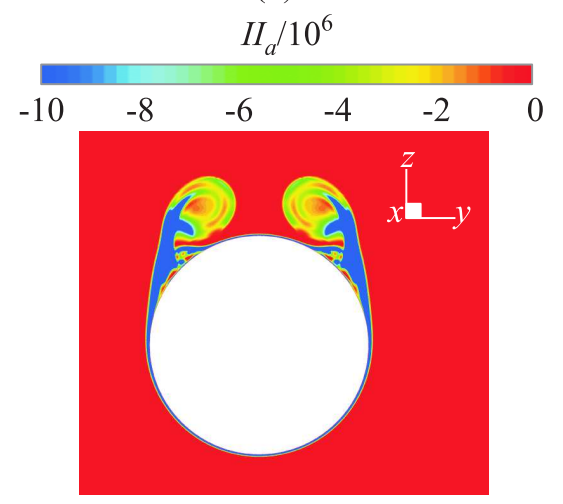

(e)

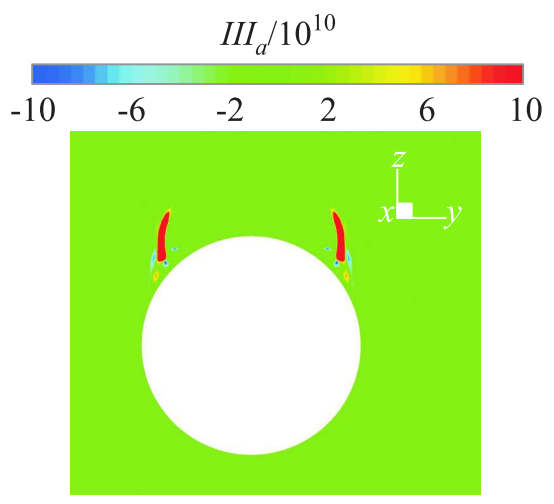

$(f)$

Figure 8 First three invariants of the stress $\left(\tau_{i j}^{*}\right)$ and anisotropy $\left(a_{i j}^{(\mathrm{ex})} \rho k\right)$ tensors of EARSM around the main vortex $(x=0.77 \% L)$ at $\operatorname{Re}=4.2 \cdot 10^{6}, \mathrm{M}=0.15$, and $\alpha=20^{\circ}$ 

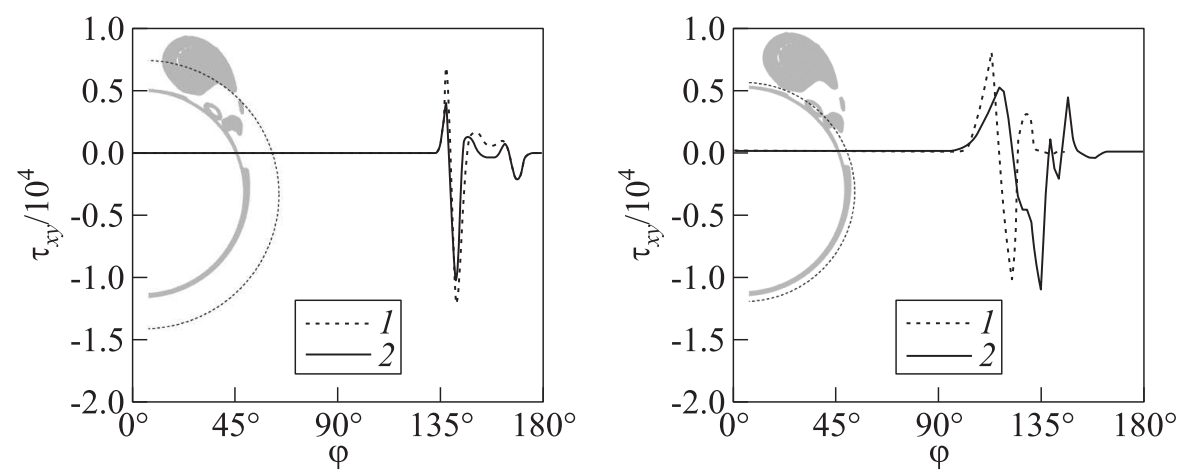

(a)
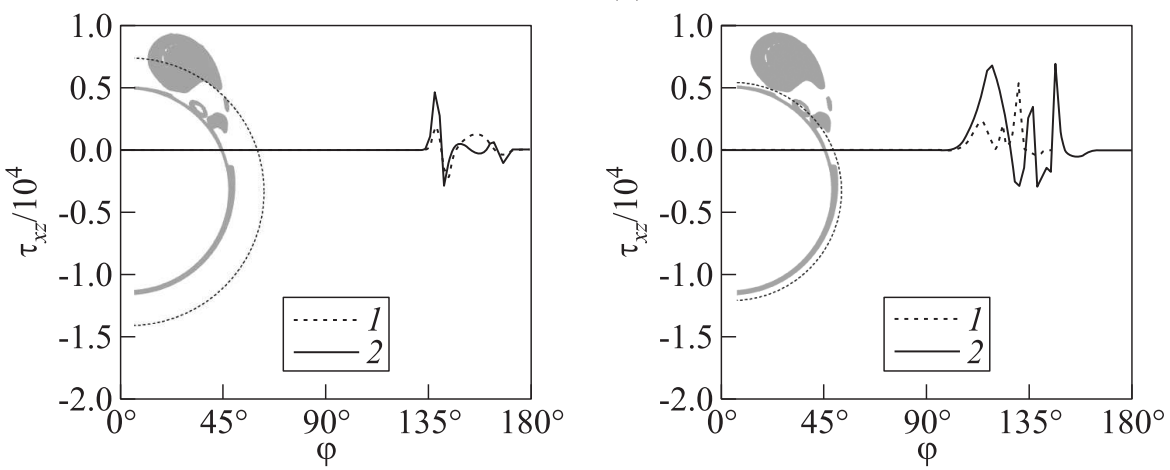

(b)
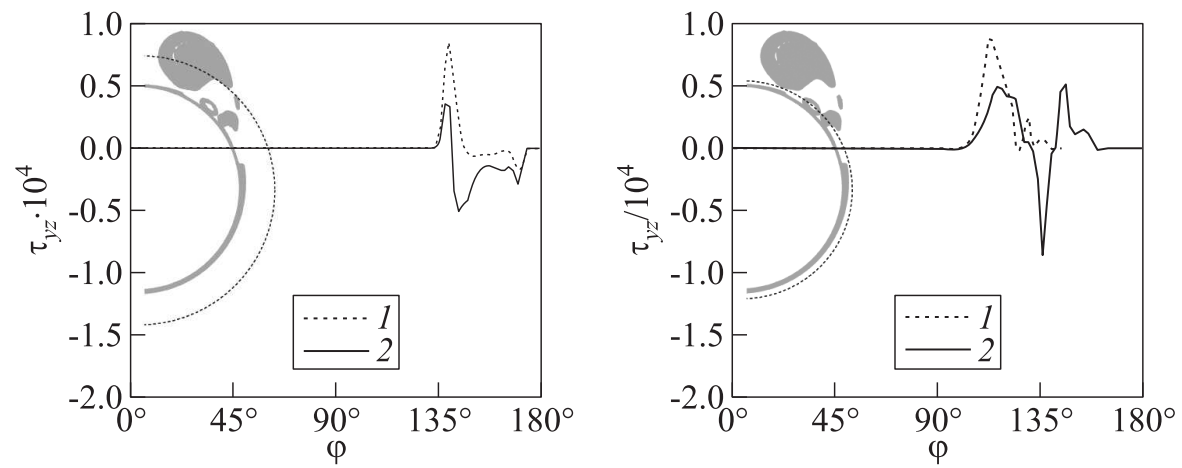

(c)

Figure 9 Comparison of stress tensor components $\left(\tau_{i j}\right)$ between $k-\omega$ SST $(1)$ and $k-\omega$ BSL + EARSM (2) at $x=0.77 \% L, \operatorname{Re}=4.2 \cdot 10^{6}, \mathrm{M}=0.15$, and $\alpha=20^{\circ}$ : (a) $\tau_{x y} ;(b) \tau_{x z} ;$ and $(c) \tau_{y z}$ 


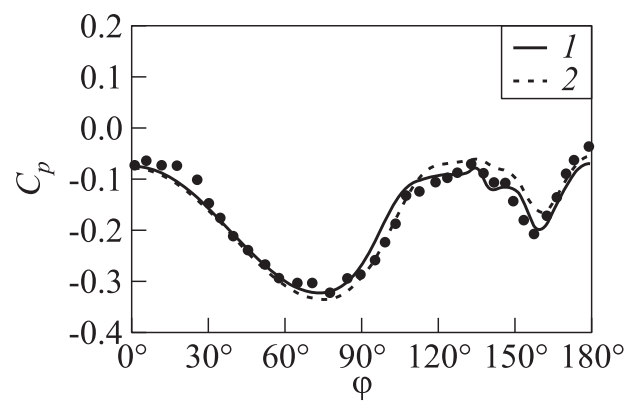

(a)

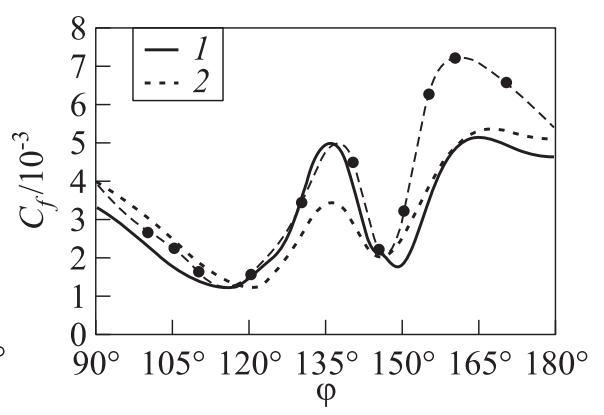

(b)

Figure 10 Comparison of $C_{p}(a)$ and $C_{f}(b)$ for URANS (1) and SAS $\left(2, \Delta t^{*}=0.02\right.$ and 0.005$)$ at $x=0.77 \% L, \operatorname{Re}=4.2 \cdot 10^{6}, \mathrm{M}=0.15$, and $\alpha=20^{\circ}$. Curves refer to calculations and signs to experiments

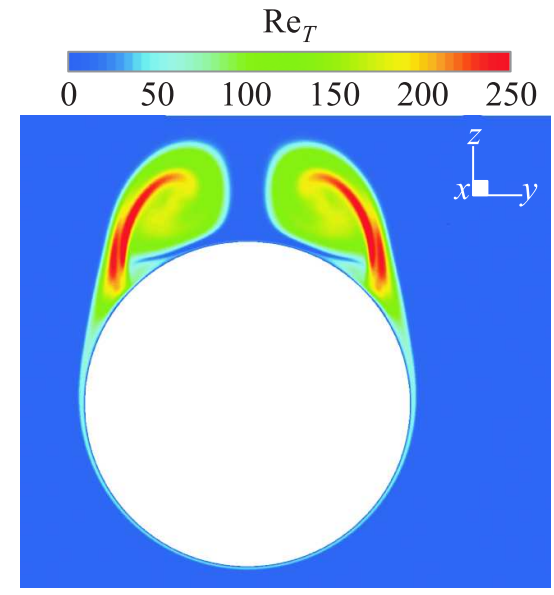

(a)

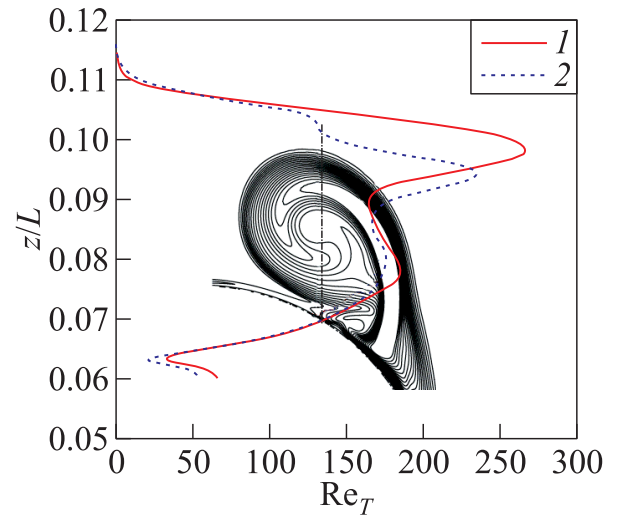

(b)

Figure 11 Turbulent eddy viscosity $\left(\operatorname{Re}_{T}\right)$ through a slice at $x=0.77 \% L$, Re $=4.2 \cdot 10^{6}, \mathrm{M}=0.15$, and $\alpha=20^{\circ}:$ (a) SAS-SST; and (b) line through the vortex; isolines of $\operatorname{Re}_{T}$ are shown ( 1 - URANS-EARSM; and 2 - SAS-SST)

\subsection{Analysis of the Flow}

Figure 12 shows contours of $Q$-criterion [37] at different spanwise sections along the spheroid and a detail at $x=77 \% \mathrm{~L}$. Friction over the spheroid is also included. As can be observed, a main vortex is generated located at $160^{\circ}$ of azimuth. The secondary vortex is also captured, located at an azimuth of $140^{\circ}$, 


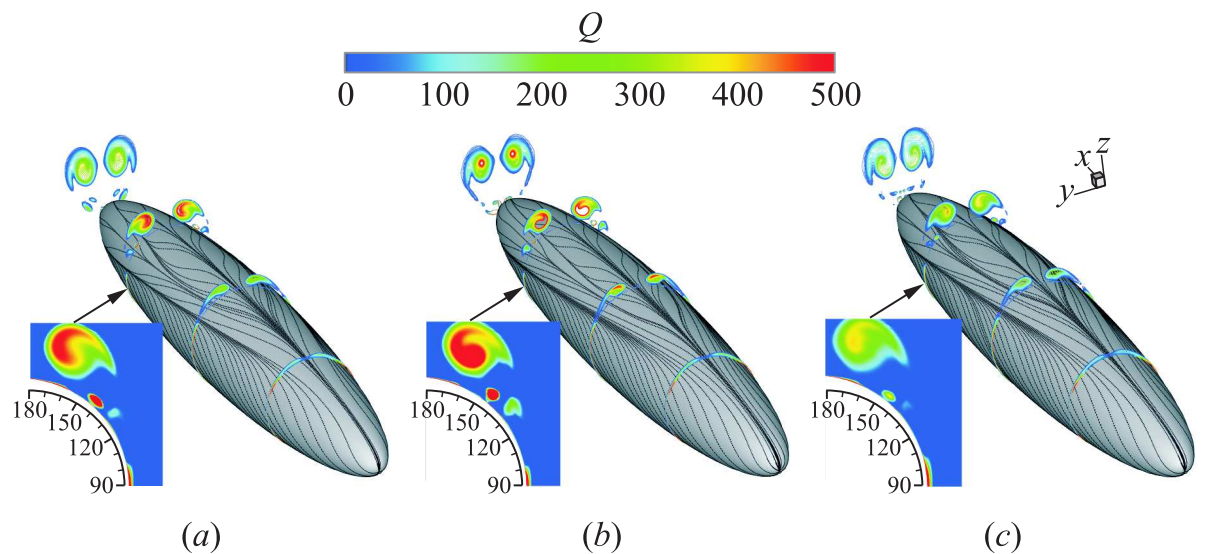

Figure 12 Contours of $Q$-criterion [37], with detail at $x=77 \% L$; $\operatorname{Re}=4.2 \cdot 10^{6}$; $\mathrm{M}=0.15$; and $\alpha=20^{\circ}:(a) k-\omega \mathrm{SST} ;(b) k-\omega \mathrm{BSL}+\mathrm{EARSM}$; and (c) SAS-SST

which is in good agreement with experiments [13]. The third vortex is also present at $130^{\circ}$, but is much weaker than the other two. As the EARSM model produces less eddy viscosity at the vortex core, the vortices are tighter and less diffused at their edge than with the $k-\omega$ SST or the SAS-SST models. This will lead to a stronger interaction of vortices at the rear of the spheroid. The SASSST model presents lower levels of $Q$-criterion as a result of the overall reduction of eddy viscosity throughout the flow-field.

In Fig. 13, the contours of helicity $(h)$ on a slice at $x=77 \% \mathrm{~L}$ are presented. Helicity is obtained by the dot product of the velocity and the vorticity vectors, $h=\boldsymbol{U} \cdot(\nabla \times \boldsymbol{U})$. Good agreement with experiments [13] is observed. As can be seen, the secondary vortex is further detached from the wall when the EARSM is employed. Likewise, lower levels of helicity in the secondary vortex are predicted by the SAS-SST model.

Friction lines projected onto a plane are shown in Fig. 14. A close view zoom of a region on the upper surface (from $90^{\circ}$ to $180^{\circ}$ of azimuth) is also included that extends from mid-length to the rear of the spheroid. As can be observed, the primary separation line $(A)$ is practically identical in both URANS solutions $\left(\varphi=105^{\circ}\right.$ at $\left.77 \% L\right)$. Conversely, the SAS-SST model predicts the primary separation at higher azimuth angle $\left(\varphi=105^{\circ}\right.$ at $\left.77 \% L\right)$. There are differences between all three models in the secondary separation line $(B)$.

The EARSM predicts a line that begins at $55 \% \mathrm{~L}$ and stays at $150^{\circ}$. The SST model predicts a later separation (from $65 \% L$ ) and moves the separation line inboards from $140^{\circ}$ to $150^{\circ}$ further downstream. For the SAS model, it also starts from $65 \% L$ but in this case, stays at approximately $150^{\circ}$. This earlier 


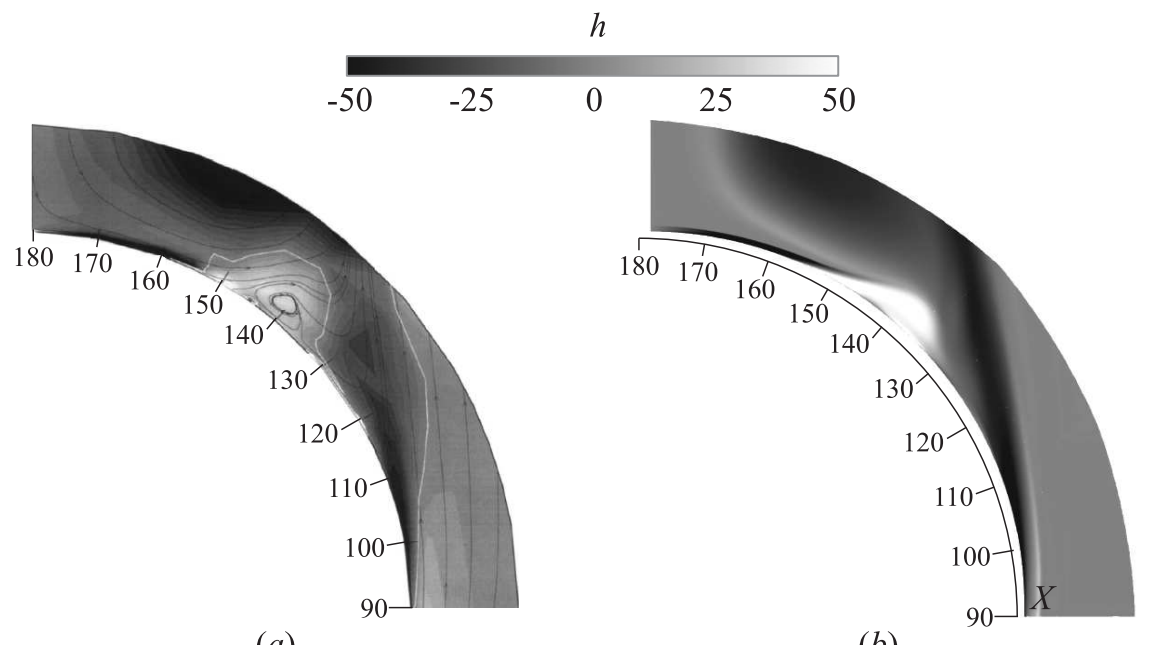

(a)

(b)

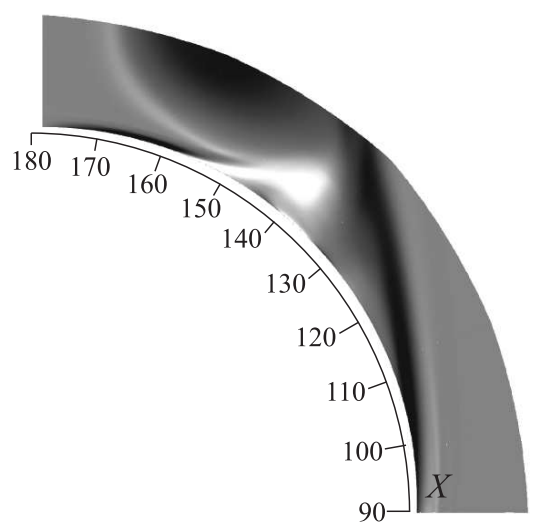

(c)

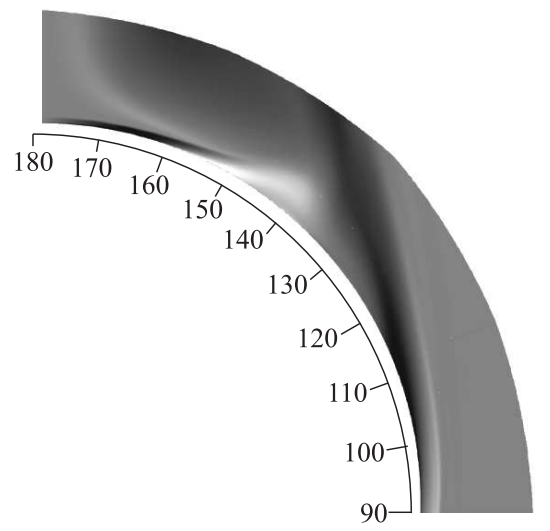

(d)

Figure 13 Contours of helicity through a slice at $x=77 \% L, \operatorname{Re}=4.2 \cdot 10^{6}, \mathrm{M}=0.15$, and $\alpha=20^{\circ}:(a)$ experiments [13]; (b) $k-\omega \mathrm{SST}$; $(c) k-\omega$ BSL + EARSM; and $(d)$ SASSST

secondary separation predicted by the EARSM was also reported by Morrison et al. [24]. Very small differences are observed for the reattachment line $(C)$ between the URANS solutions, while the SAS model predicts a line at later azimuth.

In Fig. 15, the primary and secondary separation lines location is validated against experiments [13]. These lines were measured using constant temperature and constant current anemometry and using oil flow visualization. It should 


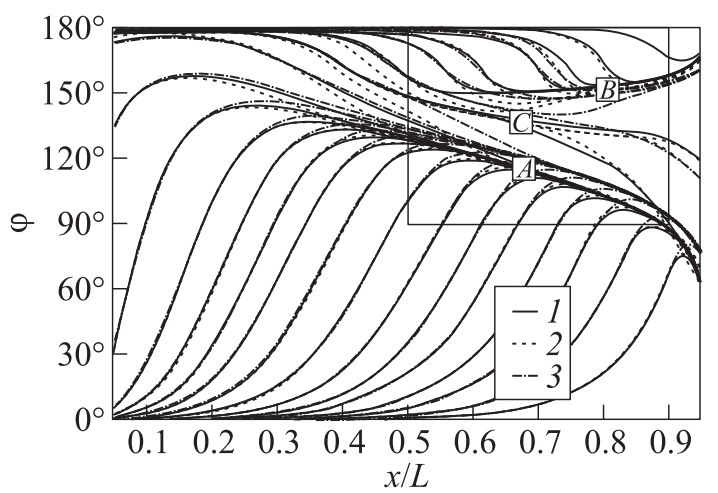

(a)

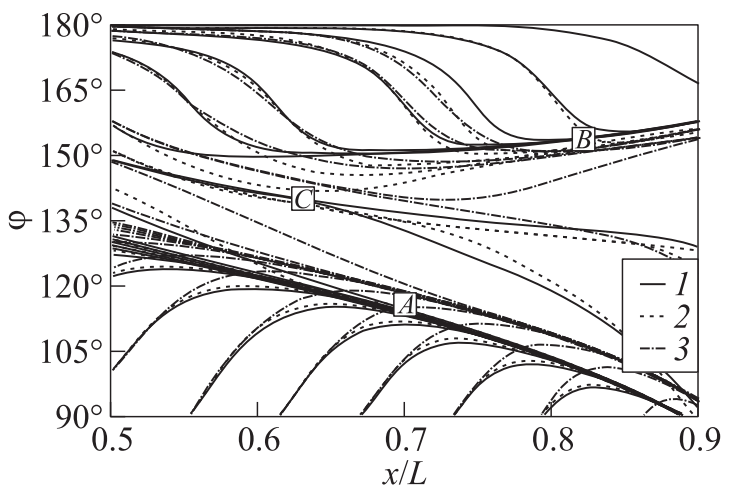

(b)

Figure 14 Friction lines on the surface of the spheroid at $\mathrm{Re}=4.2 \cdot 10^{6}, \mathrm{M}=0.15$, and $\alpha=20^{\circ}$ : (a) full view; (b) $\varphi=90^{\circ}-180^{\circ}$ region; $1-k-\omega$ BSL + EARSM; $2-$ $k-\omega \mathrm{SST}$; and 3 - SAS-SST

be noted that in the experiments, the boundary layer was tripped at $20 \% \mathrm{~L}$. In the present CFD results, a fully turbulent flow was assumed. Differences between experiments and CFD from 0 to $30 \% L$ are expected and results should be only compared from $30 \% \mathrm{~L}$. Similar trends between CFD and experiments are observed and the best agreement for both primary and secondary lines is obtained between the EARSM and the oil flow measurements.

\subsection{Effect of Transition}

In this subsection, transition effects for the spheroid pitched at $20^{\circ}$ are studied with the $k-\omega-\gamma-\operatorname{Re}_{\theta}$ transition model of Menter [35]. 


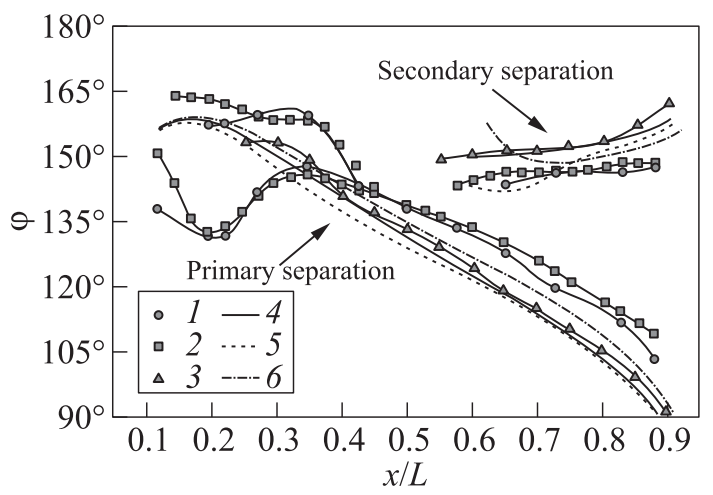

Figure 15 Comparison between CFD (curves) and experiments [13] (signs) for the primary and secondary separation lines at $\operatorname{Re}=4.2 \cdot 10^{6}, \mathrm{M}=0.15$, and $\alpha=20^{\circ}: 1$ constant temperature anemometry; 2 - current anemometry; 3 - oil flow; $4-k-\omega$ $\mathrm{BSL}+\mathrm{EARSM} ; 5-k-\omega \mathrm{SST}$; and $6-\mathrm{SAS}-\mathrm{SST}$

Figure 16 presents isosurfaces of the turbulent flow region (where $\operatorname{Re}_{T}>1$ ) for the fully turbulent and transitional cases (tests SPH.3 and SPH.4 of Table 1, respectively). For the later, the solutions at three instances within one flow travel time are provided. Instance $t_{1}$ corresponds to a phase of $\psi=0^{\circ}$ of the oscillation of the transition boundary, instance $t_{2}$ corresponds to a phase of $120^{\circ}$, and instance $t_{3}$ corresponds to $\psi=240^{\circ}$. The case where the SAS-SST model was used in Fig. 16 a clearly shows a fully turbulent flow. As can be seen in Fig. 16b, most of the lower surface of the spheroid remains laminar if the $\gamma-\operatorname{Re}_{\theta}$ is employed. In addition, due to the large pitch angle and the surface curvature, the turbulent region moves from an azimuth angle of $180^{\circ}$ at $5 \% \mathrm{~L}$ to $95^{\circ}$ at $50 \% \mathrm{~L}$ and $60^{\circ}$ at $95 \% \mathrm{~L}$. However, this transition boundary does not stay fixed with time as shown in Fig. $16 b$.

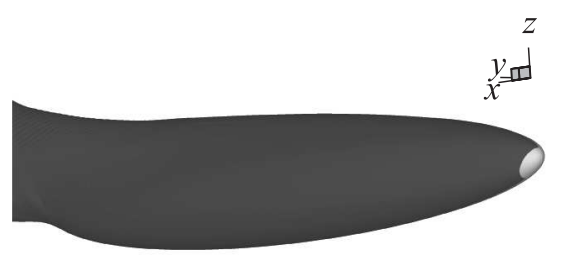

(a)

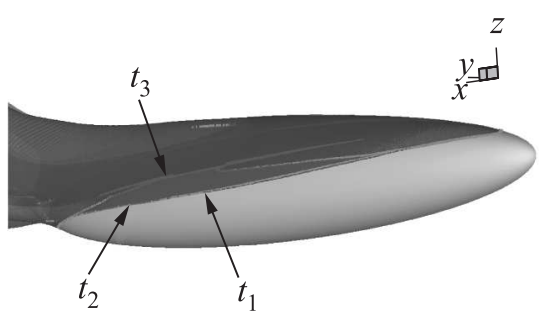

(b)

Figure 16 Comparison of turbulent region (isosurfaces of $\operatorname{Re}_{T}>1$ ) between a fully turbulent (SAS-SST) $(a)$ ) and a transition modeling (transitional flow $\left(\gamma-\operatorname{Re}_{\theta}\right)$ at three instances) $(b)$ cases at $\operatorname{Re}=4.2 \cdot 10^{6}, \mathrm{M}=0.15$, and $\alpha=20^{\circ}$ 


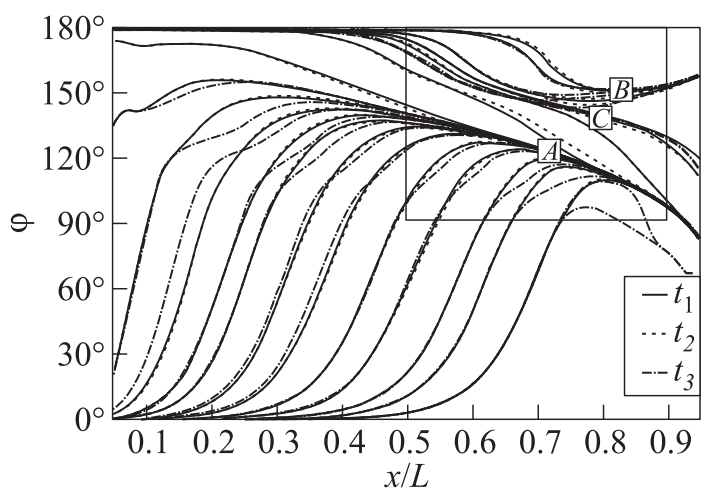

(a)

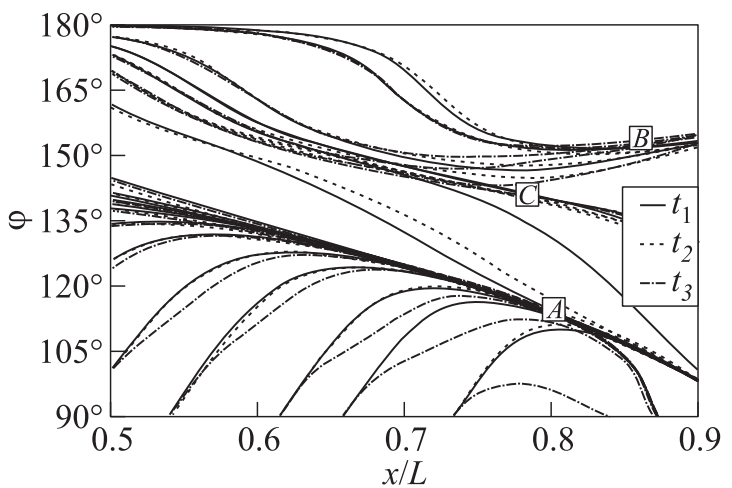

(b)

Figure 17 Friction lines on the spheroid surface, using a transition model at Re $=4.2 \cdot 10^{6}, \mathrm{M}=0.15$, and $\alpha=20^{\circ}$ : (a) full view; and $(b) 90^{\circ}-180^{\circ}$ region

It should be noted that unlike the previous cases, the separation and reattachment lines are also not fixed with time.

Figure 17 presents surface friction lines at three instances within one flow travel time. As can be observed, the primary separation line $(A)$ stays practically the same from nose to $80 \% \mathrm{~L}$. However, at instance $t_{3}$, the streamlines change curvature and the separation line from $80 \% \mathrm{~L}$ to the rear change azimuth angle from $80^{\circ}$ to $70^{\circ}$. The secondary separation line $(B)$ stays around $150^{\circ}$, but oscillates with $\pm 5^{\circ}$ of amplitude. Compared to the fully turbulent cases (see Fig. 14), this secondary separation line is located much further downstream. Finally, the reattachment line $(C)$ is similar at all instances.

This unsteadiness affects the location of the vortices. For the same instance within one travel time $\left(t_{3}\right)$, Fig. $18 a$ shows the variations in $C_{f}$ on a slice through $x=77 \% \mathrm{~L}$. Azimuth angles of $0^{\circ}$ to $90^{\circ}$ correspond to the lower surface and 


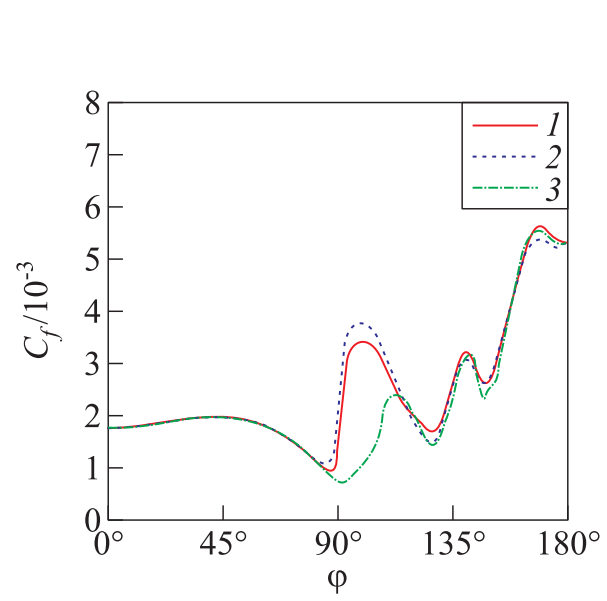

(a)

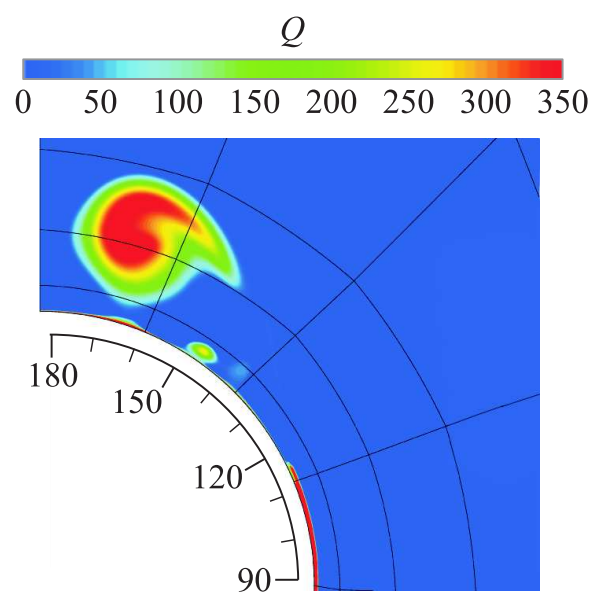

(b)

Figure 18 Oscillation of the vortices within a travel time, predicted by the transition model (slice at $x=0.77 \% L$ ) at $\operatorname{Re}=4.2 \cdot 10^{6}, \mathrm{M}=0.15$, and $\alpha=20^{\circ}:(a) C_{f}$ oscillation $\left(1-t_{1} ; 2-t_{2} ;\right.$ and $\left.3-t_{3}\right)$; and $(b)$ vortex at instance $t_{3}$

the boundary layer was predicted as laminar. In this region, the skin friction remained constant. The fully turbulent region (from $150^{\circ}$ to $180^{\circ}$ ) also stayed constant through time. Oscillations are observed in the region in-between (from $90^{\circ}$ to $150^{\circ}$ ) due to the oscillations of the transition location with time. This is the result of the expansion and contraction of the main vortex. As can be seen in Fig. 18b, the main and secondary vortices moved closer to the spheroid surface at instance $t_{3}$. These results show the importance of fixing the transition point to fully characterize the flow around this type of shapes.

\subsection{Effects on the Loads}

Table 2 provides a summary of the drag and lift for the studied cases. Note that the coefficients are nondimensionalized taking the URANS $k-\omega$ SST as reference. Since an oscillatory pattern was observed in the transitional case, the loads presented for this case were averaged. Taking the URANS $k-\omega$ SST as baseline, the total drag predicted by the EARSM increased by $3 \%$ which was due to an increase in the pressure drag, while the friction drag stayed practically the same. Using the SAS-SST formulation, the total drag was reduced by $7.5 \%$. However, the predicted friction drag in this case was higher and the pressure drag lower than for the URANS solution. The transition model predicted a much lower overall drag $(-34 \%)$, where both the $C_{d_{p}}$ and $C_{d_{f}}$ were reduced. This 
Table 2 Drag and lift for the spheroid at $20^{\circ}$ of pitch

\begin{tabular}{lcccc}
\hline \multicolumn{1}{c}{ Turbulence model } & $C_{d_{p}}$ & $C_{d_{f}}$ & $C_{d}$ & $C_{l}$ \\
\hline URANS SST & 1.00 & 1.00 & 1.00 & 1.00 \\
URANS BSL + EARSM & 1.04 & 1.00 & 1.03 & 1.02 \\
SAS SST & 0.86 & 1.10 & 0.92 & 0.87 \\
URANS $\gamma-\operatorname{Re}_{\theta}$ & 0.66 & 0.65 & 0.66 & 0.67 \\
\hline
\end{tabular}

reduction was expected, as a large laminar region was predicted with this model. Subtile differences in lift are observed between fully turbulent cases. The SASSST model provided the lowest lift values. Conversely, the transitional model provided a much lower lift (22\% lower than SAS-SST).

These results show that although there is substantial difference in the vortical structures predictions and how these affect the pressure and friction on the spheroid's surface, the overall loads are not affected as much for the fully turbulent cases. The effect of transition conversely is very important and highly affects both lift and drag predictions.

\subsection{Application Case - Bare Hull of a Hybrid Air Vehicle}

The analysis on spheroids showed the differences between turbulence models. As an application example, the bare hull of a HAV approximating the Airlander of Hybrid Air Vehicles Ltd. at model scale (see Fig. 1b) is studied here.

\subsubsection{Analysis of turbulent quantities}

Figure 19 shows the contours of turbulent eddy viscosity on a slice at $80 \% \mathrm{~L}$. As can be observed, at $20^{\circ}$ of pitch, the EARSM slightly reduces the turbulence levels. This is better observed in Fig. $20 a$ that shows a comparison of $\operatorname{Re}_{T}$ on lines passing trough the main vortex. Note that the shaded vortices included in this figure are the contour lines of $Q$-criterion. These small differences between models are due to the fact that at this pitch angle, the vortices are not fully rolled up. If the pitch angle is increased to $30^{\circ}$, the vortices are fully developed and the EARSM behaves similarly to the spheroid cases. The eddy viscosity at the vortex core is in this case highly reduced, as can be seen in the contours in Fig. $19 b$ (right column) and in the lines through the main vortex in Fig. $20 b$.

\subsubsection{Analysis of the flow-field}

Slices with contours of $Q$-criterion along the HAV are provided in Fig. 21, with a detail of the flow at $80 \% \mathrm{~L}$. At this axial station, only the main and sec- 


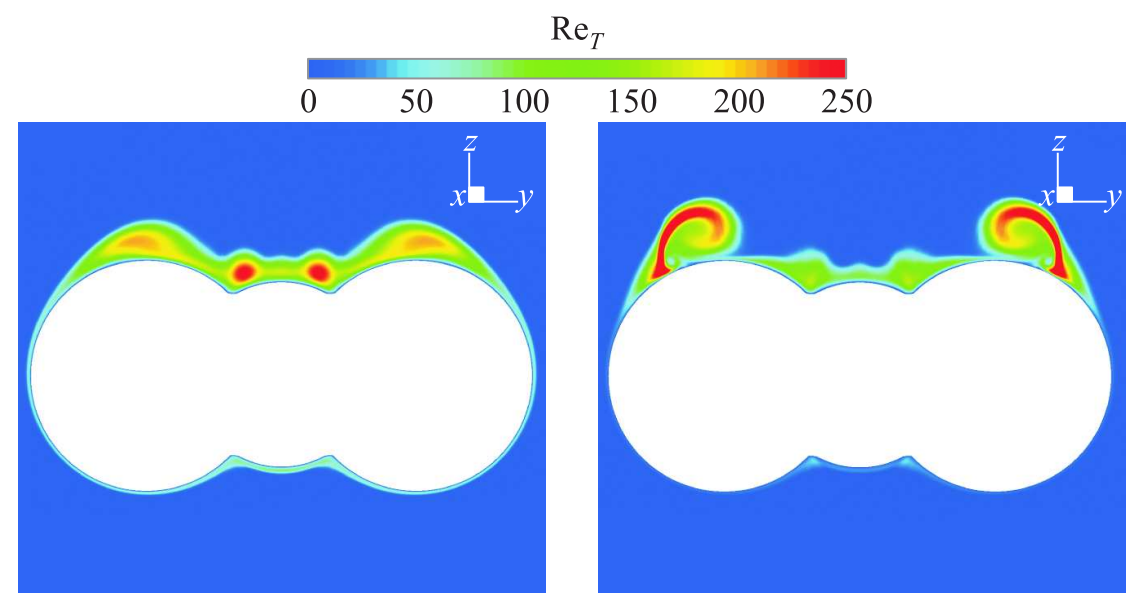

(a)

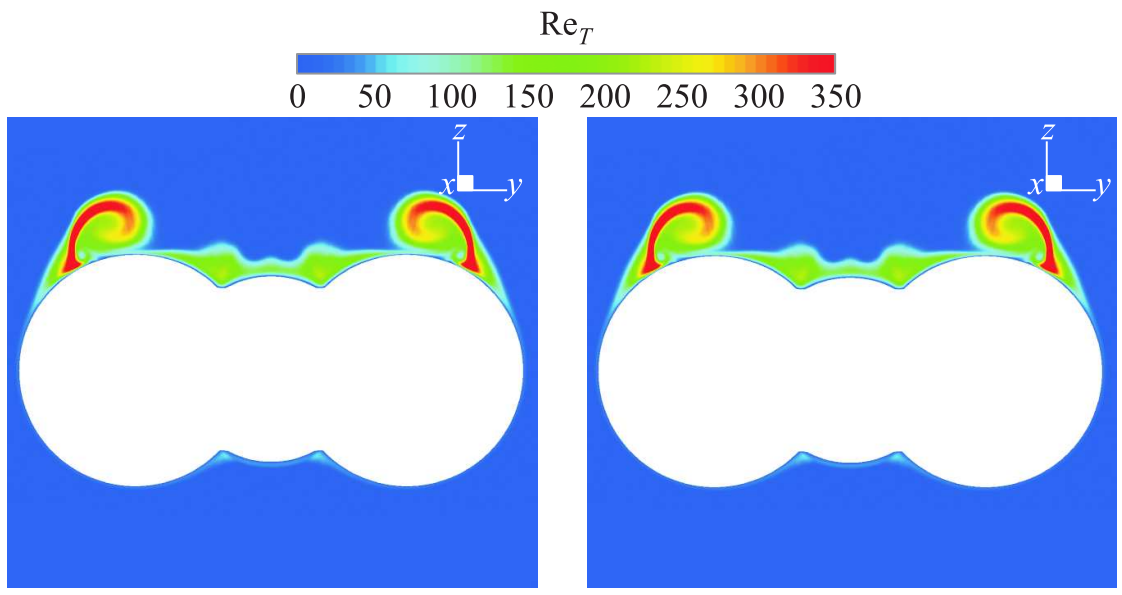

(b)

Figure 19 Turbulent eddy viscosity $\left(\operatorname{Re}_{T}\right)$ through a slice at $x=0.8 \% L, \operatorname{Re}=3 \cdot 10^{6}$, and $\mathrm{M}=0.12$ : $(a) \alpha=20^{\circ} ;(b) \alpha=30^{\circ}$; left column $-k-\omega \mathrm{SST}$; and right column $k-\omega$ BSL + EARSM

ondary vortices are present for the $k-\omega$ SST model. The main vortex is centered at $\varphi=180^{\circ}$ of azimuth for the SST model and at $170^{\circ}$ for the EARSM. The secondary vortex is located at $\varphi=155^{\circ}$ for both models. The EARSM captures the third vortex at $145^{\circ}$ of azimuth as can be seen in Fig. $21 b$. In addition, the vortices predicted by the EARSM are much tighter and stronger due to their lower eddy viscosity at the core. Compared to the spheroid flow, shown in Fig. $12 b$, the vortices generated around the HAV are located at $20^{\circ}$ 


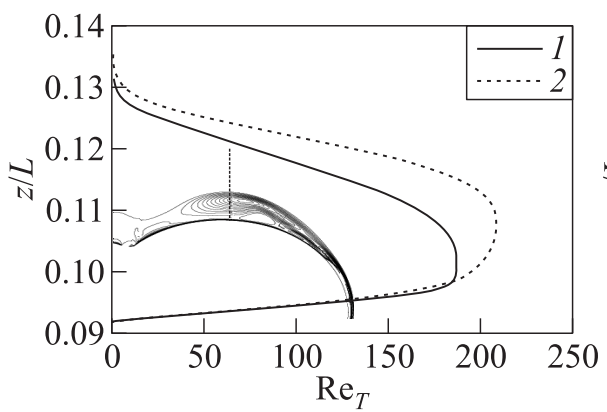

(a)

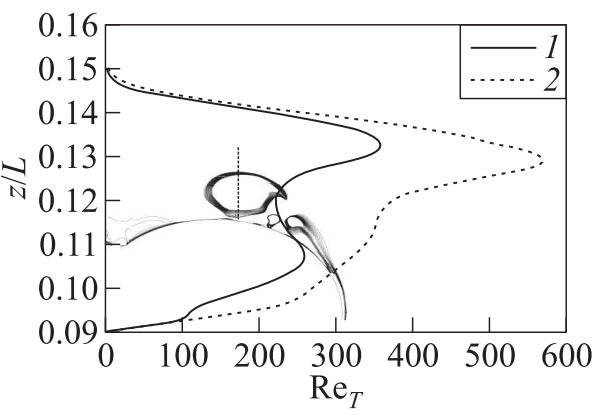

(b)

Figure 20 Turbulent eddy viscosity $\left(\mathrm{Re}_{T}\right)$ on a line passing through the main vortex $(x=0.8 \% L)$ at $\operatorname{Re}=3 \cdot 10^{6}, \mathrm{M}=0.12$, and $\alpha=20^{\circ}(a)$ and $30^{\circ}(b): 1-k-\omega$ $\mathrm{BSL}+\mathrm{EARSM}$; and $2-k-\omega \mathrm{SST}$. Isolines correspond to $Q$-criterion [37]

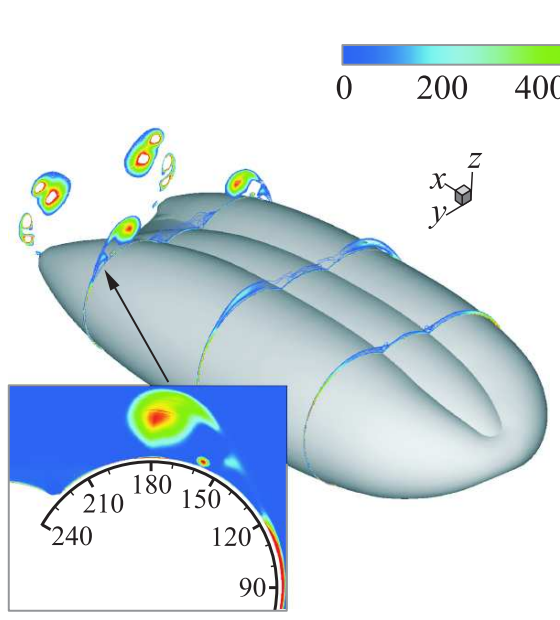

(a)
$Q$
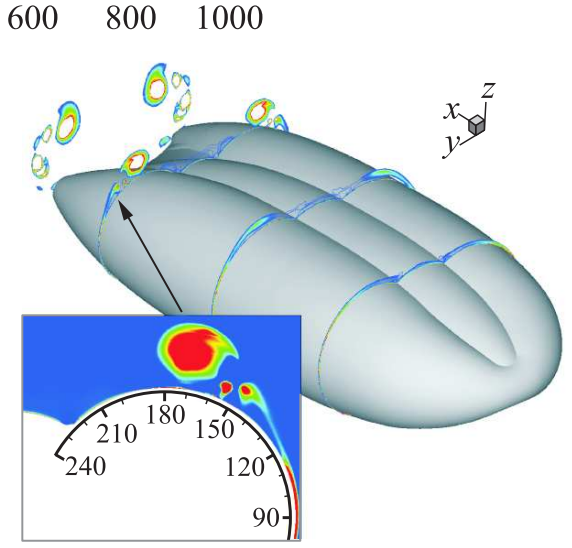

(b)

Figure 21 Contours of $Q$-criterion through a slice at $x=0.8 \% \mathrm{~L}$ at $30^{\circ}$ of pitch, $\mathrm{Re}=3 \cdot 10^{6}$, and $\mathrm{M}=0.12:(a) k-\omega \mathrm{SST}$; and $(b) k-\omega \mathrm{BSL}+\mathrm{EARSM}$

of azimuth higher. Since the surface of the airship is much wider, the vortices can develop more freely, while in the spheroid, they are confined in a smaller region.

Friction lines projected onto a 2D plane are presented in Fig. 22. Only one separation line $(A)$ on the upper surface is observed at $20^{\circ}$ of pitch starting 


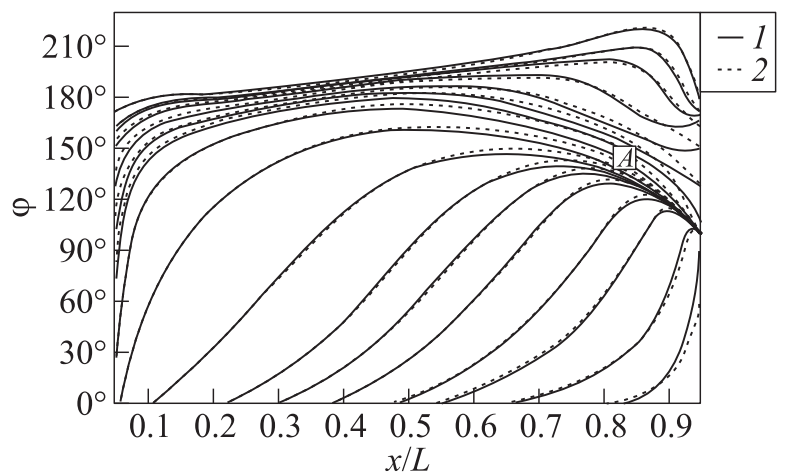

(a)

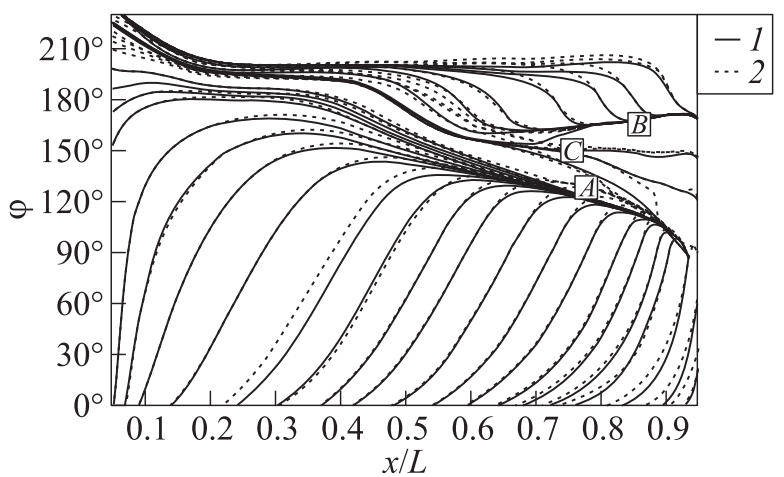

(b)

Figure 22 Friction lines projected onto a $2 \mathrm{D}$ plane at $\mathrm{Re}=3 \cdot 10^{6}, \mathrm{M}=0.12$, and $\alpha=20^{\circ}(a)$ and $30^{\circ}(b): 1-k-\omega$ BSL + EARSM; and $2-k-\omega \mathrm{SST}$

at $70 \% \mathrm{~L}$, with the same pattern for both turbulence models. This differs from the spheroid flow that at this pitch angle had primary and secondary separation lines and a reattachment region, as seen in Fig. 14 . At $30^{\circ}$ of pitch shown in Fig. $22 b$, a similar pattern to the spheroid's is observed. There is a $3^{\circ}$ of azimuth difference between turbulence models in the location of the primary separation line $(A)$. The secondary separation $(B)$ and reattachment $(C)$ lines are predicted by both models at the same axial and azimuthal positions.

Figure 23 compares the primary and secondary separation lines between the spheroid and the HAV at $\alpha=20^{\circ}$ and $30^{\circ}$. There is very little resemblance between spheroid and HAV at $20^{\circ}$ of pitch. In the latter, only primary separation is present and starts much further downstream than for the spheroid. On the other hand, a very similar pattern is observed between spheroid and HAV at $30^{\circ}$ of pitch. It is interesting that both primary and secondary separation lines are 


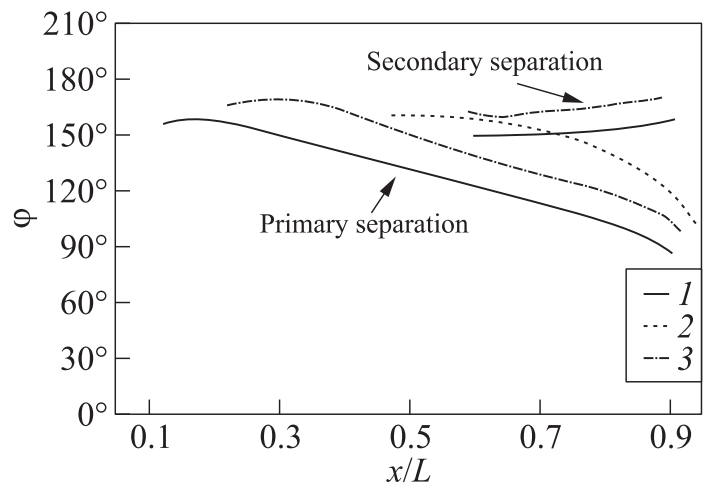

Figure 23 Primary and secondary separation lines: $1-$ spheroid $\left(\alpha=20^{\circ}\right) ; 2-$ $\operatorname{HAV}\left(\alpha=20^{\circ}\right) ;$ and $3-\operatorname{HAV}\left(\alpha=30^{\circ}\right)$

parallel, with the only difference in the fact that the HAV's lines are shifted by $20^{\circ}$ of azimuth.

\subsubsection{Analysis of the aerodynamic loads}

Figure 24 shows azimuthal surface pressure and friction distributions on a slice at $80 \% \mathrm{~L}$. Note that the analysis is focused on the outer lobe of the HAV where the vortices are developed. The layout of the vortices with isolines of $Q$-criterion is included in the figures. Azimuth $\psi=0^{\circ}$ corresponds to the lower surface, $\psi=90^{\circ}$ is on the side, and $\psi=180^{\circ}$ is the uppermost point in the upper surface. At $20^{\circ}$ of pitch, as shown in Fig. $24 a$, the EARSM model presents a dip in pressure and a high peak in friction at $\varphi=180^{\circ}$ which is an indicative of the generation of a vortex. For this case, the results were compared with a coarser grid (9 million cells) and very small differences in pressure were observed, while bigger differences are seen in the friction coefficient. The peak-to-peak values due to the presence of the secondary vortex are larger in the EARSM than in the $k-\omega$ SST model at $30^{\circ}$ of pitch as the predicted vortices are stronger, as seen in Fig. 24b. In this case, the main vortex is located at an azimuth of $\varphi=170^{\circ}$ and the secondary vortex is predicted at $\varphi=150^{\circ}$. Note that an oscillation is observed very close to the secondary peak, which is due to the presence of the third vortex very close to the second one. This phenomenon is clearly captured by the EARSM.

The drag and lift predicted by both models are finally compared in Table 3. As for the spheroid cases, the coefficients were also nondimensionalized with the $k-\omega$ SST model. At $20^{\circ}$ of pitch, there is a total increase in drag of $22 \%$ when the EARSM is employed. This is due to the contributions of the pressure and friction drag components that increased by $25 \%$ and reduced by $8 \%$, respectively. Similar 

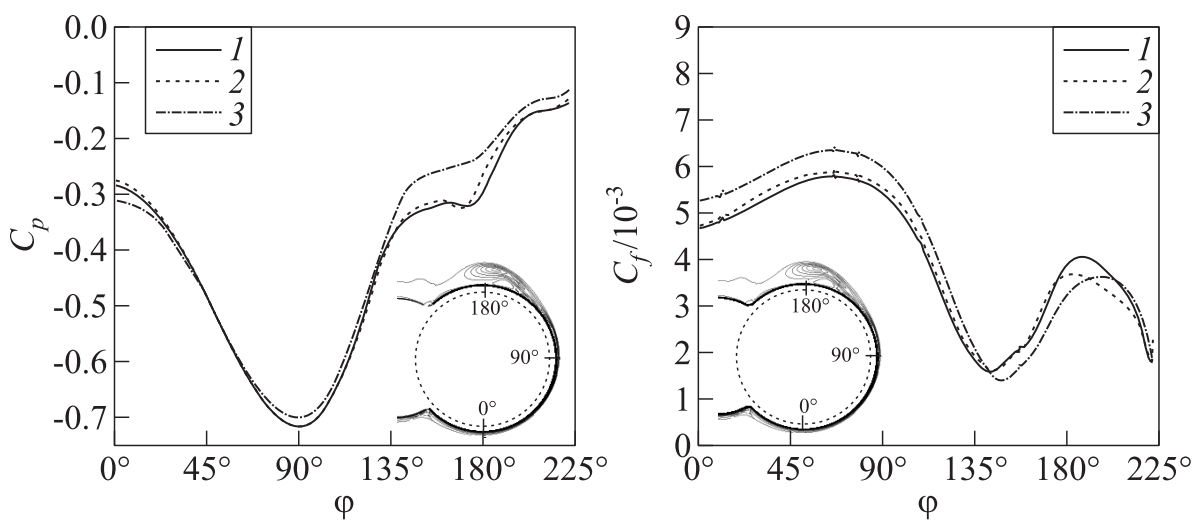

(a)
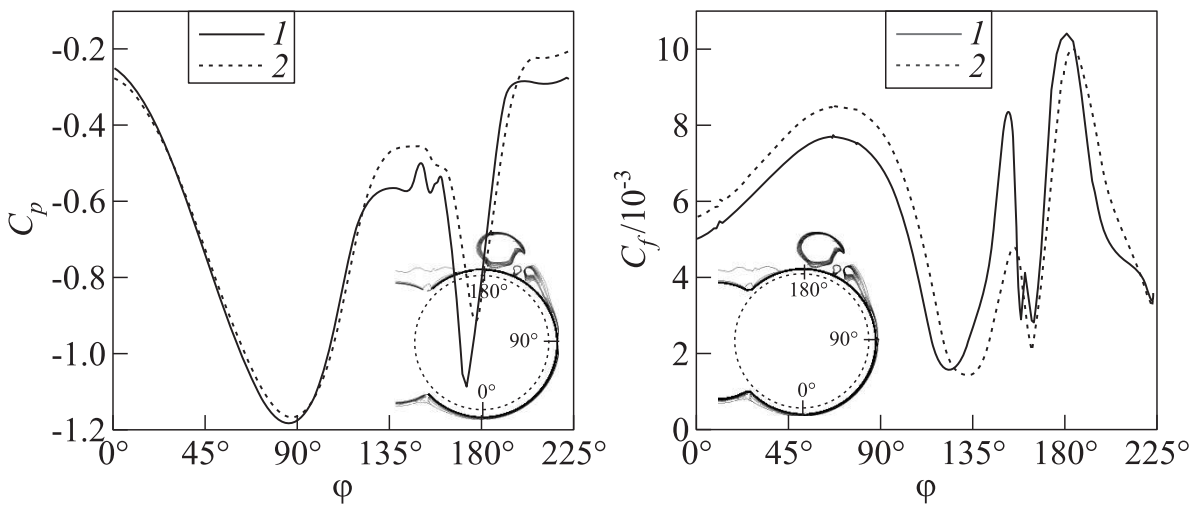

(b)

Figure 24 Comparison of pressure (left column) and friction (right column) coefficients at $x=0.80 \% \mathrm{~L}$. Isolines corresponding to $Q$-criterion [37]: $(a) \alpha=20^{\circ}(1-$ $k-\omega$ BSL + EARSM, $31 \cdot 10^{6}$ cells; $2-k-\omega$ BSL + EARSM, $9 \cdot 10^{6}$ cells; and $3-k-\omega$ SST, $31 \cdot 10^{6}$ cells); and (b) $\alpha=30^{\circ}(1-k-\omega$ BSL + EARSM and $2-k-\omega$ SST $)$

Table 3 Drag and lift for the HAV

\begin{tabular}{cccccc}
\hline Pitch angle & Turbulence model & $C_{d_{p}}$ & $C_{d_{f}}$ & $C_{d}$ & $C_{l}$ \\
\hline \multirow{2}{*}{$20^{\circ}$} & SST & 1.00 & 1.00 & 1.00 & 1.00 \\
& BSL + EARSM & 1.25 & 0.92 & 1.22 & 1.12 \\
\hline \multirow{2}{*}{$30^{\circ}$} & SST & 1.00 & 1.00 & 1.00 & 1.00 \\
& BSL + EARSM & 1.19 & 0.91 & 1.17 & 1.09 \\
\hline
\end{tabular}


behavior is observed at $30^{\circ}$ of pitch where the EARSM predicted a drag $17 \%$ higher than the SST model. An increase in the lift of $12 \%$ and $9 \%$ at pitch of $20^{\circ}$ and $30^{\circ}$, respectively, was observed.

The results for $C_{l}$ are approximately three times higher than for the spheroid. This was expected due to the fact that the HAV has three lobes instead of one and produces more lift due to its camber. Taking this into consideration, overall, similar loadings are compared between spheroid and HAV flows.

These results show that the turbulence model plays an important role in the prediction of this flow. Although in the spheroid cases no large differences in the loads were observed between the SST and the EARSM models, the HAV flows appear to be more sensitive to the employed turbulence model.

\subsection{Flow Topology Around a Complete Hybrid Air Vehicle Configuration}

This subsection aims to further analyze the flow around the complete configuration of a HAV. A comparison is first shown between the AL $\mathbf{4}$ and AL $\mathbf{5}$ cases of Table 1 . The pressure coefficient $\left(C_{p}\right)$ at the symmetry and mid-planes are presented in Fig. 25. The solutions are practically the same, with small differences close to the back of the vehicle. Grid convergence can, therefore, be assumed, with the employed URANS framework. As can also be observed in Fig. 25, favourable pressure gradients $(\partial p / \partial s<0)$ are present from the nose to $25 \% L$, which indicates fully attached flow. From $25 \% L$ to $80 \% L$, a neutral pressure gradient $(\partial p / \partial s \approx 0)$ is present on both upper and lower surfaces. From $80 \% L$, the adverse pressure gradient $(\partial p / \partial s>0)$ leads to flow separation.

The location of the transition onset on the AL body is estimated, employing empirical criteria and fully turbulent CFD results. For this, the Michel [38] and

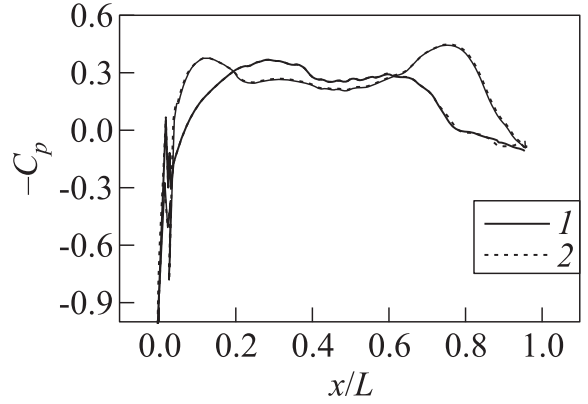

(a)

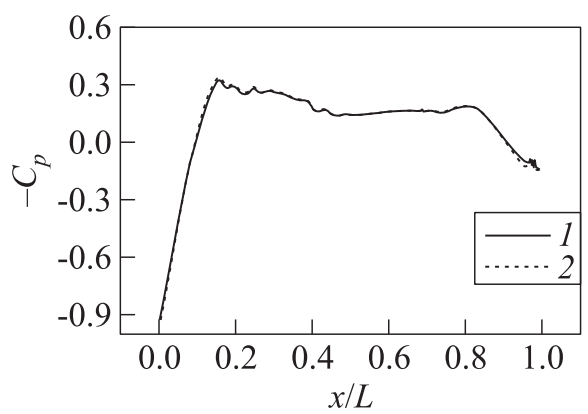

(b)

Figure 25 Effect of the mesh density in $C_{p}$ at the symmetry plane $(a)$ and at the mid-plane $(b)$ of the approximated HAV: $1-\mathbf{A L} 4$; and $2-\mathbf{A L} \mathbf{5}$ 
Cebeci and Smith criteria [39] are employed on streamlines extracted inside the boundary layers of the flow solutions. These criteria are based on experimental data on flat plates and aerofoils and provide an estimate of the location of the transition onset when the local momentum thickness Reynolds number $\left(\operatorname{Re}_{\theta, t r}\right)$ reaches a particular value. In the case of Michel's criterion [38], the transition onset location takes place when

$$
\operatorname{Re}_{\theta, \mathrm{tr}} \approx 2.9 \operatorname{Re}_{x, \mathrm{tr}}^{0.4}
$$

where $\operatorname{Re}_{x, \text { tr }}$ is the Reynolds number based on the distance measured from the stagnation point. In Cebeci and Smith's correlation [39], the transition is located at the point where

$$
\operatorname{Re}_{\theta, \mathrm{tr}}=1.174\left(1+\frac{22400}{\operatorname{Re}_{x, \mathrm{tr}}}\right) \operatorname{Re}_{x, \mathrm{tr}}^{0.46}
$$

Figure 26 shows the momentum thickness Reynolds number $\left(\operatorname{Re}_{\theta}\right)$ along a streamline of the CFD solution and the empirical transition criteria where changes in Reynolds number were considered. At $\operatorname{Re}=3 \cdot 10^{6}$, the presence of favourable pressure gradients make the boundary layer to stay laminar until approximately $80 \% \mathrm{~L}$. When the Reynolds number is reduced, the boundary layer becomes less turbulent. The opposite effect happens when the Reynolds number increases. The transition onset is moved downstream and upstream for lower

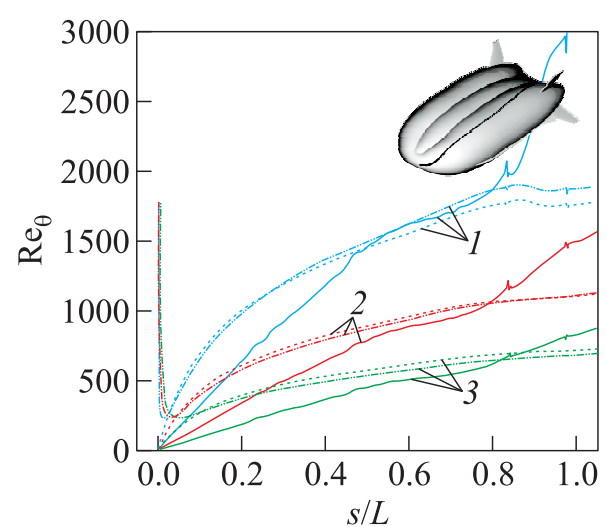

(a)

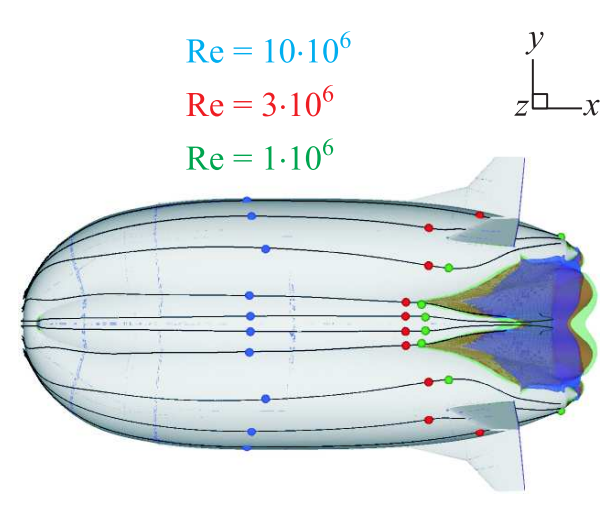

(b)

Figure 26 Sensitivity of the transition onset to the Reynolds number: $(a)$ empirical transition criteria $\left(1-\operatorname{Re}=10 \cdot 10^{6} ; 2-\mathrm{Re}=3 \cdot 10^{6} ; 3-\mathrm{Re}=1 \cdot 10^{6}\right.$; solid curves refer to CFD solution; dotted curves to Michel's criterion [38]; and dashdotted curves to Cebeci and Smith's criterion [39]); and (b) transition onset and isosurfaces of reverse flow 
and higher Reynolds, respectively. When the Reynolds number was reduced by a third, the transition onset was moved downstream by approximately $5 \% \mathrm{~L}$.

When Re was increased from $3 \cdot 10^{6}$ to $10 \cdot 10^{6}$, the transition onset moved upstream by $42 \% \mathrm{~L}$. The results show the strong effect of the Reynolds number to the nature of the boundary layer. As can be seen in the isosurfaces of the reversed flow in Fig. 26b, when the Reynolds number is higher and, therefore, the upstream boundary layer is more turbulent, the region of the separated flow is smaller. Conversely, at lower Reynolds number, the flow tends to separate more.

\subsection{Study of the Role of the Components on a Complete Hybrid Air Vehicle Configuration}

To assess which components are the ones contributing the most to the overall lift and drag, Fig. 27 shows the relative changes with respect to the bare hull case (AL 1) for the 4 vehicle configurations presented in Figs. $2 d$ and 3 . At $10^{\circ}$ of pitch, as presented in Fig. $27 a$, the fins increased the lift by more than twice and the LERX and strakes contributed to a further small increase. The drag also increased when each component was considered, but in less percentage. As Fig. $27 b$ shows, at $20^{\circ}$ of pitch, the increase in loads is not as drastic as in the previous case. The fins increased the lift by less than $50 \%$ and the strakes seem to provide more lift than the LERX. In this case, the penalties in drag were closer to the increase in drag than in the previous case. These results show that with the presented configuration of the approximated HAV, the fins are the components that contribute the most to the lift and, also, drag.

Regarding the stability of the vehicle, the pitching moment $\left(C_{M_{y}}\right)$ and the change of it with the pitch angle $\left(C_{M_{\alpha}}\right)$, calculated at the center of the volume, are presented in Fig. 28. Positive $C_{M_{\alpha}}$ means nose up attitude and negative is

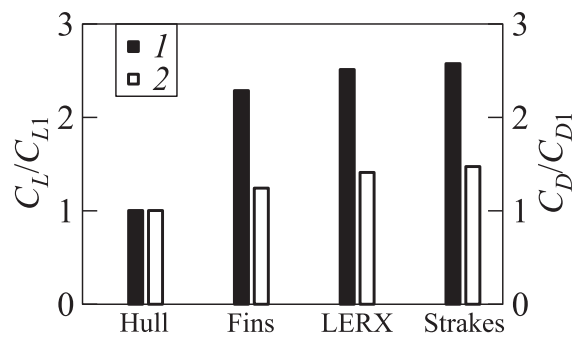

(a)

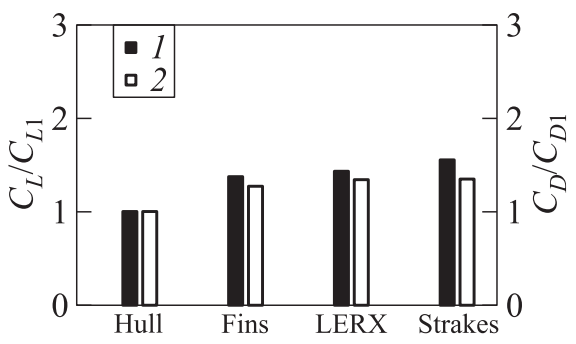

(b)

Figure 27 Contribution of each component to the lift (1) and drag (2) with respect to the bare hull configuration: $(a) \alpha=10^{\circ}$; and $(b) \alpha=20^{\circ}$ 


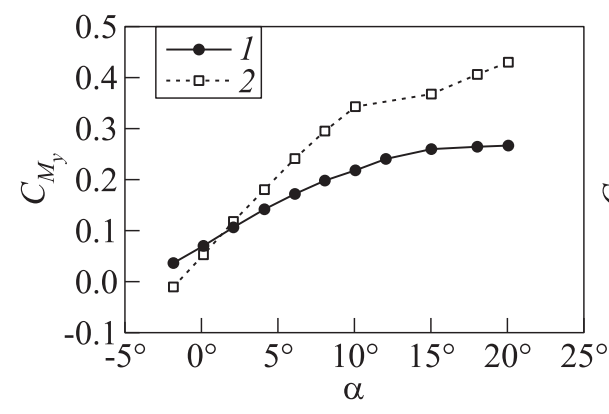

(a)

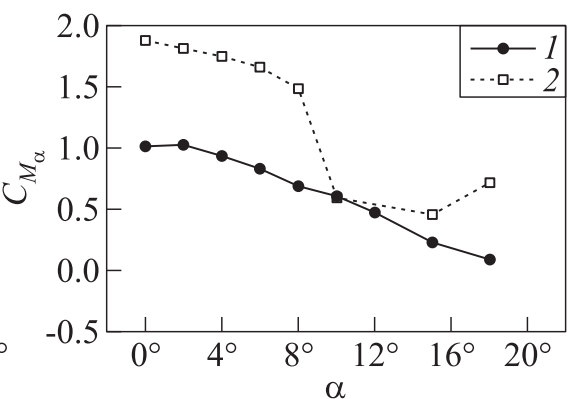

(b)

Figure 28 Pitching moment $\left(C_{M_{y}}\right)(a)$ and derivative $\left(C_{M_{\alpha}}\right)$ of pitching moment $(b)$ at the center of the volume: 1 - full vehicle; and 2 - bare hull

nose down. As Fig. $28 b$ shows, both bare hull and full configurations seem to be unstable as the tendency of the vehicle is to pitch up for positive changes in pitch. This should be attributed to the fact that no buoyancy force was included in the computations. Similar behavior was observed in other works in the literature that did not account for buoyancy effects. This is the case of the experiments on the AKRON [40], YEZ-2A [41], and ZHIYUAN-1 [42,43] airships that also presented positive $C_{M_{\alpha}}$.

Nevertheless, the presence of the aerodynamic components has a stabilizing effect, since there is a reduction in the pitch derivative. This was also seen by Wang et al. [42] and Freeman [40]. In addition, since the 'AL' is not axisymmetric, at zero pitch angle, the pitching moment is not zero which differs from more traditional airship configurations [41-44].

\section{CONCLUDING REMARKS}

The HMB2 solver was validated for the flow around a 6:1 prolate spheroid. Within the URANS framework, the $k-\omega$ SST and the $k-\omega$ EARSM turbulence models were compared with the experimental data available in the open literature. Both models showed very good agreement in the pressure predictions. Regarding the friction coefficients, the best agreement was obtained with the EARSM due to a lower production of eddy viscosity and better resolution of the vortices. This was observed in a comparison of the turbulent levels that showed a strong reduction of turbulence in the core of the vortices predicted by the EARSM. Analyses of the components of the stress tensor showed that the EARSM seemed to capture more spatial variations than the $k-\omega$ SST model. 
The investigation followed by a comparison between the URANS-EARSM and SAS-SST models. The best predictions were also obtained with the URANSEARSM, showing that to properly predict spheroid-type flows, accounting for turbulent anisotropy is needed. Further, flow-field analyses were performed for these models. Contours of $Q$-criterion revealed clear differences in the shape and size of the vortices, with the EARSM vortices predicted tighter and stronger. This was also observed in helicity contours. Surface friction lines showed an identical primary separation line for both URANS models, but the SAS-SST model predicted the separation at a higher azimuth angle. In addition, the EARSM predicted an earlier secondary separation line. Transition effects were also investigated and it was found that the location of the transition region oscillated in time which directly affected the pressure and skin friction predictions.

The effect of the turbulence model on the overall loads was also presented. Differences of $4 \%$ to $6 \%$ were observed in the fully turbulent cases, being the EARSM the one that predicted the highest drag. As expected, the transition model predicted much less drag (a reduction of about 30\%) due to the presence of a large laminar region on the spheroid. However, for better assessment of turbulence models, experiments with integrated loads and free-transition measurements for spheroid flows are needed.

The analysis of turbulence models finished with the bare hull of a HAV. In this case, similar behavior to the spheroid's was observed once the vehicle was pitched at $30^{\circ}$. Like in spheroid flows, the vortices were better predicted with the EARSM. The overall loads, conversely, were much more sensitive to the turbulence model in the airship case than in the spheroid.

The paper finished with the analysis of the flow around an early design of a HAV where the body vortices were identified. Due to the presence of favourable pressure gradients, the onset of transition was predicted close to the rear of the airship, at $80 \% \mathrm{~L}$. Sensitivity studies on the Reynolds number showed a further downstream transition onset for lower Reynolds numbers and an increase of Reynolds led to an earlier transition onset. The study continued by exploring the role of each component of the airship on the aerodynamic coefficients and the stability derivatives. The results showed that with the standard vehicle configuration, the fins contributed the most to an increase in lift and also drag.

\section{ACKNOWLEDGMENTS}

The results were obtained using the EPSRC funded ARCHIE-West High Performance Computer (www.archie-west.ac.uk), EPSRC grant No. EP/K000586/1. The N8 HPC facility "Polaris" at Leeds University and the Chadwick cluster of the University of Liverpool were also used. Access to the systems is gratefully acknowledged as well as the financial support by the LOCATE Project. 


\section{REFERENCES}

1. Carichner, G. E., and L. M. Nicolai. 2013. Hybrids... the airship messiah? AIAA Lighter-Than-Air Systems Technology Conference Proceedings. Daytona Beach, FL.

2. Omari, K., E. Schall, B. Koobus, and A. Dervieux. 2004. Turbulence modeling challenges in airship CFD studies. Monografias del Seminario Matemático García Galdeano 31:545-554.

3. Omari, K., E. Schall, Y. Le Guer, and M. Amara. 2005. Numerical study of turbulent flow around a generic airship at high angle of attack. 4th Conference (International) on Computational Heat and Mass Transfer. Paris-Cachan, France.

4. Stockbridge, C., A. Ceruti, and P. Marzocca. 2012. Airship research and development in the areas of design, structures, dynamics and energy systems. Int. J. Aeronautical Space Sci. 13(2):170-187.

5. Gatski, T. B., and T. Jongen. 2000. Nonlinear eddy viscosity and algebraic stress models for solving complex turbulent flows. Prog. Aerosp. Sci. 36:655-682.

6. Alpman, E., and L. N. Long. 2005. Separated turbulent flow simulations using a Reynolds stress model and unstructured meshes. 43rd AIAA Aerospace Sciences Meeting and Exhibit.

7. Mor-Yossef, Y. 2015. Separated flow prediction around a $6: 1$ prolate spheroid using Reynolds stress models. Differential Reynolds stress modelling for separating flows in industrial aerodynamics. Ed. B. Eisfeld. Springer tracts in mechanical engineering ser. Springer International Publishing. 39-60.

8. Wetzel, T., R. L. Simpson, and C. J. Chesnakas. 1998. Measurement of threedimensional crossflow separation. AIAA J. 36(4):557-564.

9. Wallin, S., and A. Johansson. 2000. An explicit algebraic Reynolds stress model for incompressible and compressible turbulent flows. J. Fluid Mech. 403:89-132.

10. Grudestam, O., S. Wallin, and A. V. Johansson. 2005. An explicit algebraic Reynolds stress model based on a nonlinear pressure strain rate model. Int. J. Heat Fluid Flow 26:732-745.

11. Hellsten, A. 2005. New advanced $k-\omega$ turbulence model for high-lift aerodynamics. AIAA J. 43(9):1857-1869.

12. Wetzel, T. 1996. Unsteady flow over a $6: 1$ prolate spheroid. Virginia Polytechnic Institute and State University. PhD Thesis.

13. Chesnakas, C. J., and R. Simpson. 1997. Detailed investigation of the threedimensional separation about $6: 1$ prolate spheroid. AIAA J. 35(6):990-999.

14. Clarke, D. B., P. A. Brandner, and G. J. Walker. 2008. Experimental and computational investigation of flow around a $3: 1$ prolate spheroid. WSEAS Trans. Fluid Mech. 3(3):207-217.

15. Scott, N. W. 2004. Unsteady Reynolds-averaged Navier-Stokes predictions of the flow around a prolate spheroid. 42nd AIAA Aerospace Sciences Meeting and Exhibit.

16. Scott, N.W. 2005. Using detached eddy simulation and overset grids to predict flow around a $6: 1$ prolate spheroid. 43rd AIAA Aerospace Sciences Meeting and Exhibit. 
17. Xiao, Z., H. Chen, Y. Zhang, J. Huang, and S. Fu. 2006. Prediction for separation flows around a $6: 1$ prolate spheroid using hybrid RANS/LES methods. 44th AIAA Aerospace Sciences Meeting and Exhibit.

18. Vatsa, V., J. Thomas, and B. Wedan. 1989. Navier-Stokes computations of a prolate spheroid at angle of attack. J. Aircraft 26(11):986-993.

19. Constantinescu, G., H. Pasinato, Y. Wang, J. Forsythe, and K. Squires. 2002. Numerical investigation of flow past a prolate spheroid. Trans. ASME 124:904910 .

20. Karlsson, A., and C. Fureby. 2009. LES of the flow past a $6: 1$ prolate spheroid. 47th AIAA Aerospace Sciences Meeting including The New Horizons Forum and Aerospace Exposition.

21. Sorensen, N. 2009. CFD modelling of laminar-turbulent transition for airfoils and rotors using the $\gamma-\operatorname{Re}_{\theta}$ model. Wind Energy 12:715-733.

22. Vizhinho, R., J. Morgado, J. Páscoa, and M. Silvestre. 2015. Analysis of transitional flow in 3D geometries using a novel phenomenological model. Aerospace Sci. Technol. 45:431-441.

23. Rumsey, C. L., and T. B. Gatski. 2000. Recent turbulence model advances applied to multielement airfoil computations. AIAA Paper No. 2000-4323.

24. Morrison, J.H., A. G. Panaras, T.B. Gatski, and G. A. Georgantopoulos. 2003. Analysis of extensive cross-flow separation using higher-order RANS closure models. 21st AIAA Applied Aerodynamics Conference.

25. Wilcox, D. C. 1994. Simulation of transition with a two-equation turbulence model. AIAA J. 32(2):247-255.

26. Barakos,G., R. Steijl, K. Badcock, and A. Brocklehurst. 2005. Development of CFD capability for full helicopter engineering analysis. 31st European Rotorcraft Forum. Florence, Italy. Paper 91.

27. Osher, S., and S. Chakravarthy. 1983. Upwind schemes and boundary conditions with applications to Euler equations in general geometries. J. Comput. Phys. 50(3):447-481.

28. Van Leer, B. 1979. Towards the ultimate conservative difference scheme, V. A second order sequel to Godunov's method. J. Comp. Phys. 32:101-136.

29. Rieper, F. 2011. A low-Mach number fix for Roe's approximate Riemann solver. J. Comput. Phys. 230:5263-5287.

30. Carrión, M., M. Woodgate, R. Steijl, and G. Barakos. 2013. Implementation of allMach Roe-type schemes in fully implicit CFD solvers - demonstration for wind turbine flows. Int. J. Numer. Meth. Fluids 73(8):693-728.

31. Axelsson, O. 1994. Iterative solution methods. Cambridge, MA: Cambridge University Press. 668 p.

32. Menter, F. R., and Y. Egorov. 1010. The scale-adaptive simulation method for unsteady turbulent flow predictions. Part 1: Theory and model description. Flow Turb. Combust. 85:113-138.

33. Zografakis, G., and G. N. Barakos. 2008. Transition modelling for rotorcraft CFD. 34th European Rotorcraft Forum. Liverpool, U.K. P. $5 \mathrm{~b} 3$. 
34. Menter, F. R. 1994. Two-equation eddy-viscosity turbulence models for engineering applications. AIAA J. 32(8):1598-1605.

35. Menter, F. R., R. B. Langtry, R. B. Rikki, S. R. Suzen, P. G. Huang, and S. Volker. 2006. A correlation-based transition model using local variables - Part i: Model formulation. J. Turbomach. 128(3).

36. Steijl, R., and G. Barakos. 2008. Sliding mesh algorithm for CFD analysis of helicopter rotor-fuselage aerodynamics. Int. J. Numer. Meth. Fluids 58:527-549.

37. Jeong, J., and F. Hussain. 1995. On the identification of a vortex. J. Fluid Mech. 285:69-94.

38. Michel, R. 1951. Etude de la transition sur les profils d'aile, etablissement d'un critere de determination du point de transition et calcul de la trainee de profil incompressible. Technical Report 1/1578A.

39. Cebeci, T., and A. M. O. Smith. 1974. Analysis of turbulent boundary layers. Applied mathematics and mechanics ser. New York, NY: Academic Press. Vol. 15. $404 \mathrm{p}$.

40. Freeman, H. B. Force measurements on a 1/40-scale model of the U.S.' airship 'AKRON.' Cranfield Institute of Technology. PhD Thesis.

41. Gomes, S. B. V. 1990. An investigation of the flight dynamics of airships with application to the YEZ-2A. Cranfield Institute of Technology. PhD Thesis.

42. Wang, X., G. Fu, P. D, and X. Shan. 2010. Experimental investigations on aerodynamic characteristics of the ZHIYUAN-1 airship. J. Aircraft 47(4).

43. Suman, S., S. Lakshmipathy, and R. Pant. 2013. Evaluation of assumed-transitionpoint criterion in context of Reynolds-averaged simulations around lighter-than-air vehicles. J. Aircraft 50(2).

44. Funk, P., and T. Lutz, and S. Wagner. 2003. Experimental investigations on hull-fin interferences of the lotte airship. Aerosp. Sci. Technol. 7:603-610. 